\title{
LLE Review Quarterly Report
}

Editor: R. L. Keck

(716) 275-0259

\section{January-March 1992}

Laboratory for Laser Energetics

College of Engineering and Applied Science

University of Rochester

250 East River Road

Rochester, New York 14623.1299 
This report was prepared as an account of work conducted by the Laboratory for Latser Energetics and sponsored by New York State Energy Research and Devefopment Authority. the University of Rochester, the U.S. Department of Energy. and other agencies.

Neither the above-named sponsors, nor any of their employees, makes any warrinty. expressed or implied, or assumes any legal liability or responsihility for the accuracy, completeness, or usefulness of any information, apparatus. product, or process disclosed, or represents that its use bould not infringe privalely owned ights.

Reference herein to any specific commercial product, process, or service by trade name, mark manulacturer, or otherwise, does not necessarily constitute or imply its endorsement, recommendation, or favoring by the Uniled States Govermment or any agency thereof or any other sponsor.

Results reported in the LLE Review should not be taken as necessarily final results as they represent active research. The views and opinions of authors expressed herein do not necessarily state or reflect those of any of the above sponsoring entities. 


\section{IN BRIEF}

This volume of the LLE Review, covering the period January March 1992. contains articles on the use of diffraction gratings in laser applications, and the tabrication of gratings for use in these applications. There are two articles on the use of lasers io explore fundamental physics issues and an article on the use of a wolid-state diode array' for $x$-ray imaging. Finally', the activities of the National Laser Users Facility and the GDL and OMEGA laser systems are summarized.

Highlights of the research reported in this issue are

- High-efficiency, large-aperture, high-damage-threshold diffraction gratings are now being fabricaled. The method of their production, as well as examples of their use. is described.

- A new method for the temporal shaping of laser pulses is described. The method. salled spectral beam deflection. can be extended to other applications requiring later-bcam scanning.

- A solid-slate devector array has been used for $x$-ray imaging. A number of advantages of such arrays over alternative detectors are discussed.

- The use of picosecond laser pulses to study the ionization of atoms in intense electric lields is described.

- The use of femtosecond laser pulses to study carrier dynamics in semicondustors is discussed. 


\section{CONTENTS}

\begin{tabular}{|c|c|}
\hline \\
\hline Section 1 & ADVANCED TECHNOLOGY DEVELOPMENTS ..............6 \\
\hline $1 . A$ & High-Efficiency Holographic Cratings for \\
\hline 1.13 & $\begin{array}{l}\text { High-Power Laser Systems ....................................... } \\
\text { Shaping and Measuring a Laser Pulse Using }\end{array}$ \\
\hline & Spectral Beam Deflection .............................. \\
\hline 1.8 & 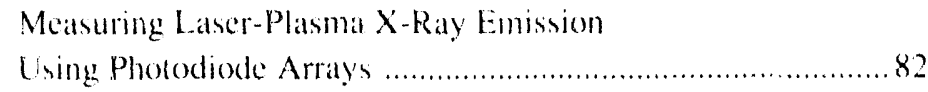 \\
\hline 1.5) & $\begin{array}{l}\text { Conization of Aioms with Intense, Linearly and } \\
\text { Circularly Polarized, Piconecond Laser Pulses...... }\end{array}$ \\
\hline 1.E & 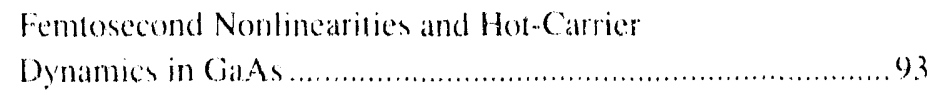 \\
\hline Section 2 & NATIONAL. LASER USERS FACILITY NEWS ................ 10. \\
\hline Section 3 & LASER SYSTEM REPOR'T ................................. \\
\hline $3 . \mathrm{A}$ & GDL. Facility Report ................ \\
\hline $3 . \mathrm{B}$ & 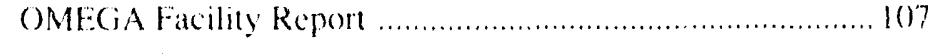 \\
\hline
\end{tabular}

PUBLICATIONS AND CONFERENCE PRLSENTATIONS 


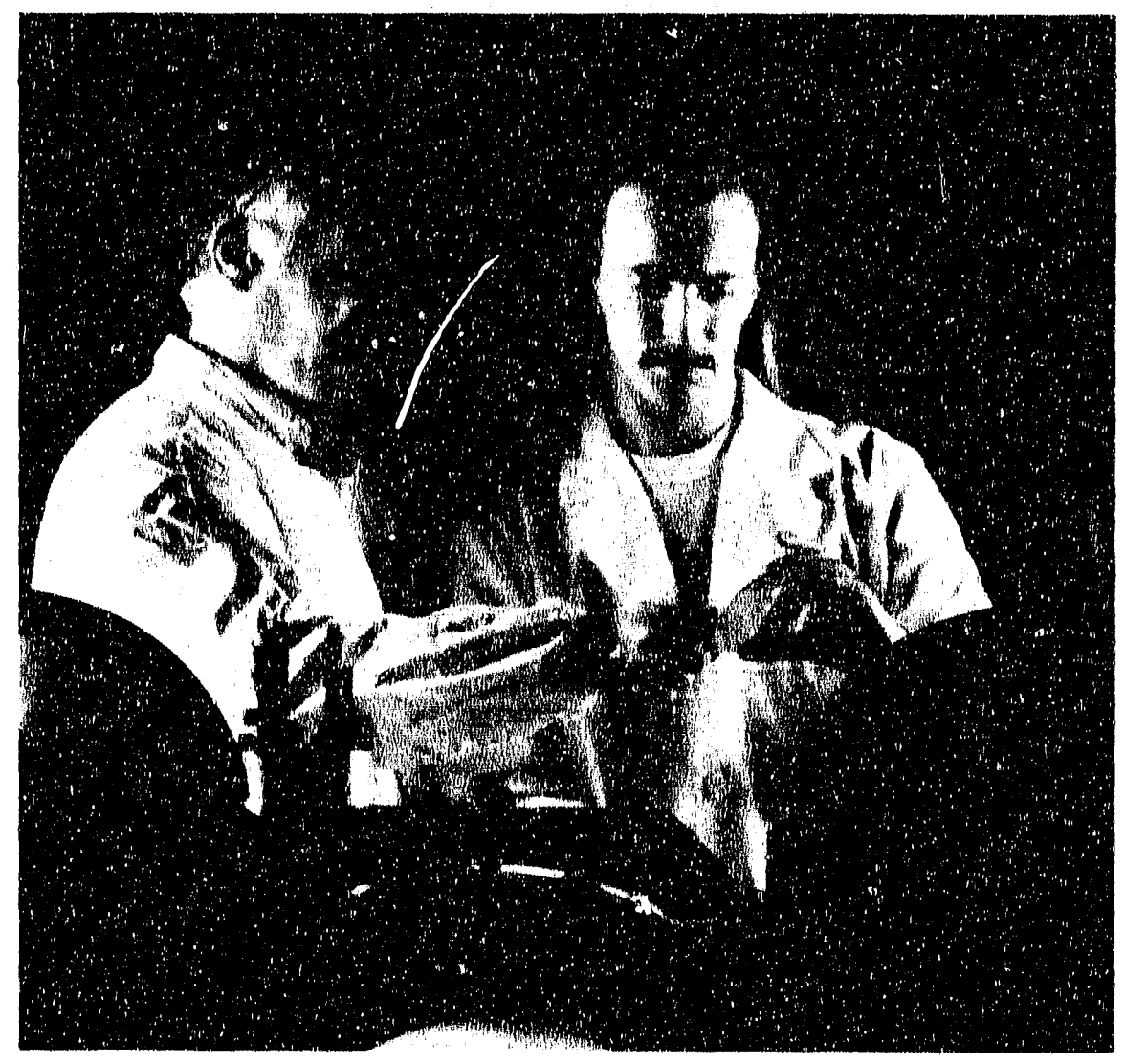

Joe Armstrotig, an Optics graduate student, shows Senior Scientist Stan Skupsky a high-power, holographic grating sample. The fabrication and use of these high-power gratings are described in two articles in this issue. 


\title{
Section 1
}

\section{ADVANCED TECHNOLOGY DEVELOPMENTS}

\section{A High-Efficiency Holographic Gratings for Iligh-Power Laser Systems}

\begin{abstract}
Large-aperture holographic transmission gratings that possess high diffraction efficiency and high damage thresholds have been designed, fabricated, and experimentally tested within high-power, solid-state laser systems. The dispersive properties of diffraction gratings provide a variety of important and interesting techmiques to control the spatial and temporal characteristics of laser light. Novel approaches to laser-bearn smoothing and pulse shaping involve the transter of information between time and space by means of the angular spectral dispersion (ASD) and latcral time delay imposed by a diffacsion grating onto a propagating laser beam. The surface-relief transmission grating. composed of periodic and contiguous grooves, provides a source of ASD and posseses the intrinsically high damage threshold required for use in high-power laser sy sems. Transmission gratings have been employed in the driver line of the OMEC iA laser system for several years without occurrence of laser-induced damage. Improved phaseconversion techniques, necessary to reach ultra-uniform levels of irradiation uniformity on the OMEGA Upgrade laser system, require the use of largeaperture transmission gratings. In addition, novel pulse-compression, pulseexpansion, and pulse-shaping schemescan now deploy highly dispersive gratings.
\end{abstract}

\section{Theoretical Modeling}

The holographic transmission grating, based on surface relief of a transparent material. deffracts an incident beam of light into various directions according to the sarne diffraction-grating equation as pertains to the holographic volume and reflection gratings or conventionaliy ruled gratings. The generalized grating equation is' 


$$
d \mid \sin (\theta)-\sin (i)]=m \lambda
$$

where $d$ is the groove spacing. $\theta$ and $i$ are the diffracted and incident angles, respectively, $m$ is the diffaction order, and $\lambda$ is the wavelength of light. Figure 5). 1 shows an enlargement of a section of the surface relief. The periodic thictiness variation constiutes a surface relief whose groove spacing and relief height (h) are typically of the order of the wavelength of light.

Fig. 5(1).

A periodic thickness variation constitutes a surface relief that cian diffract an incoming beam of light into an alternate direction. Holographic transmission gratings possess a groche spacing and surface-relief height typically of the order of the wavelength of light.

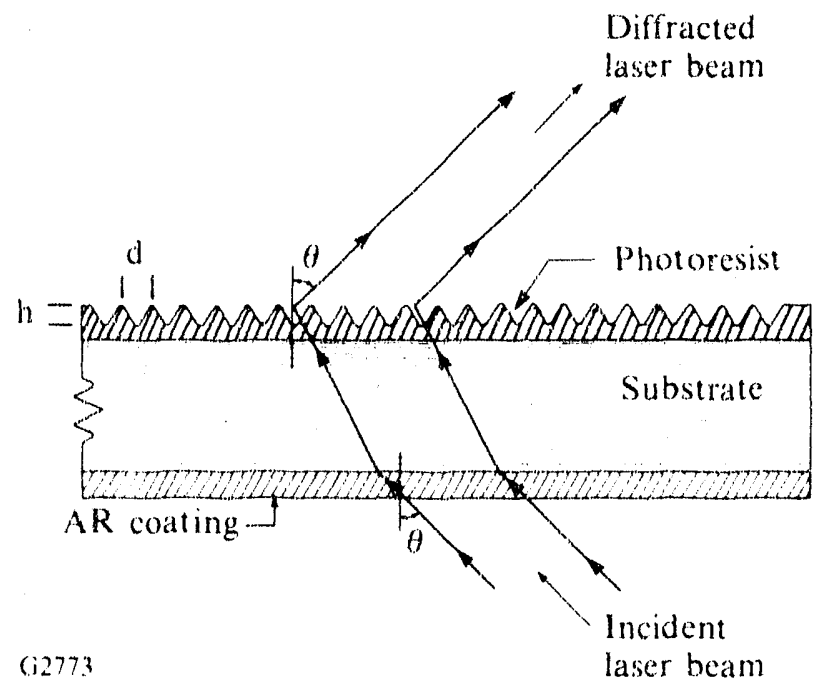

The rate of change of the diffaction angle with respect to change in wavelength is referred to as the angular dispersion ( $\Gamma$ ) of the grating and is a measure of the angular spreading of the spectral components of light. Angular dispersion is given by

$$
\Gamma=m / d \cdot \cos (\theta) .
$$

For the ypecial case of unit lateral magnification between the input beam and the output beam, where the incident and diffracted angles are equal but opposite. the expressions for the grating equation and angalar dispersion become

$$
2 d \cdot \sin (\theta)=\min
$$

and

$$
\Gamma=2 \cdot \operatorname{lan}(\theta) / \lambda
$$

respectively. These equations describe the ray directions of spectral components, but do not contain information about the efficiency with which the diliraction orcurs. 
The diffaction characteristics of a dielectric grating have been the subject of much research. Numerical solutions have been obtained for gratings of arbitrary profiles by the integral-equation and differential-equation methods. 2.3 The differential-equation methods involve either a normal mode exparision or a coupled-wave expansion. Each represents a large system of equations and is difficult tohandlecomputationally. The integral method ${ }^{4}$ provides computational case and has shown good agreement with experimental results for those gratings fabricated in the past. Theoretical predictions. ${ }^{5}$ using an integral-equation code, show that peak diffraction efficiency near unity can be achieved over a wide range of groove spacings, or design angles, as shown in Fig. 50.2(a). For each design angle the peak diffraction efficiency is obtained at a particular value for the groove aspect ratio $h / d$, which is determined by the temporally integrated energy reaching the photoresist. Figure 50.2(b) illustrates the dependence of diffraction efficiency on the aspect ratio of the grooves. This curve points out the importance of obtaining uniform and accurate exposures, since changes in groove aspect ratio cause changes in diffraction efficiency.

(a)

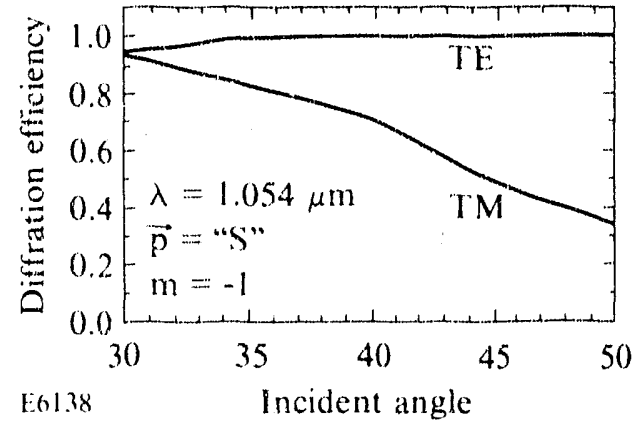

(b)

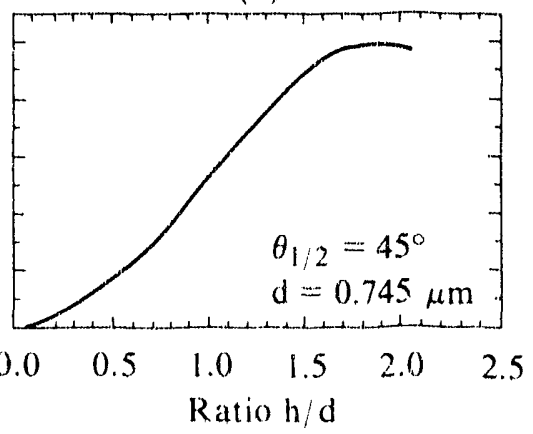

Fig. 50.2

Theoretical predictions |Fig .50.2(a) / show that the peak diffraction afficioncy approaches unity over a wide range of groove spacings or design angles. Diffaction efficiency varies with the aspect ratio of the grooves [fig. 50.2(b)].

\section{Interferometer Design}

The holographic interferometer, as shown in Fig. 50.3, consists of a laser source, beam-conditioning optics, and two beamlines that intersect at the final recording plane. Several new and unique design features contribute to its overall capability for producing high-visibility interference fringes and a deep-grooved surlace relief.

The laser is a Spectra-Physics 2045E argon ion laser, enuipped with an intracavity etalon. highly dispersive cavity mirrors, and electronic feedback control to maintain both beam power and beam centering. At a $28-\mathrm{W}$ output power in the visible, this laser is capable of $7-W$ output for simultaneous lasing of all of its ultraviolet lines. Selection of a single longitudinal mode within the $\lambda=.364 \mathrm{~nm}$ spectral line, by using an intracavity etalon and a Fabry-Perot spectrum analyzer, further restricts the max imum output power o approximately $\mathrm{IW}$. The laser is located on a separate table to prevent vibrations caused by the cooling system from reaching the interferometer. Vibrations of this type degrade fringe visibility and must be reduced as much as possible. 


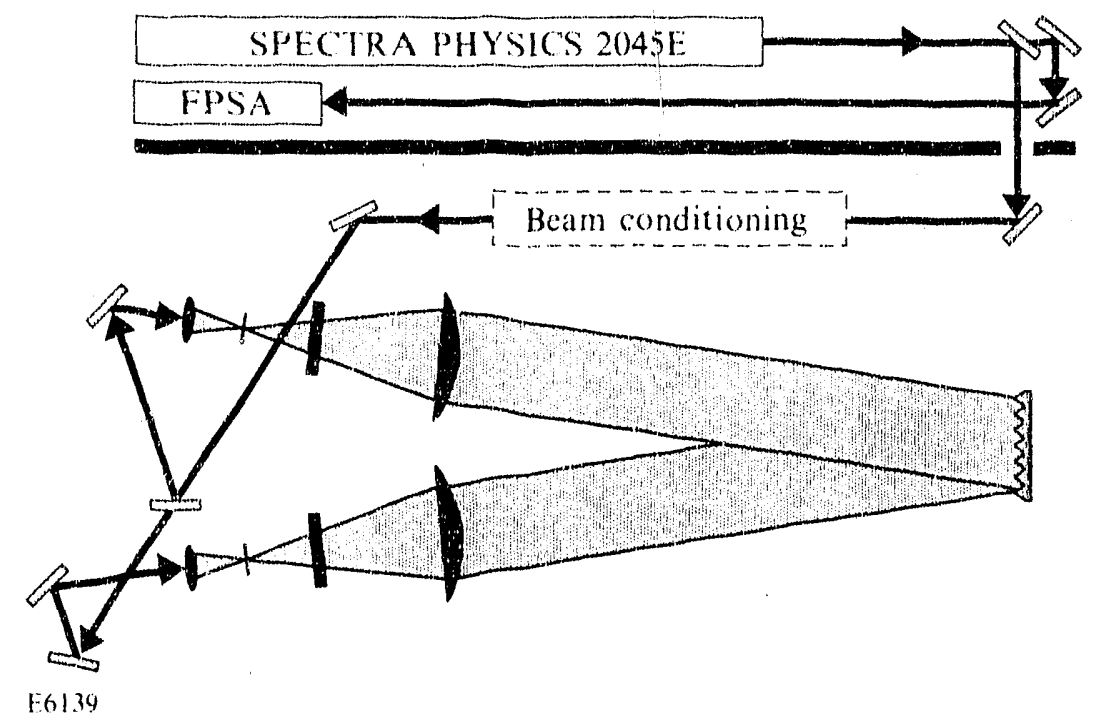

Fig. 50.3

A beam scanner, a normal-incidence beam splitter, and an even mirror configuration are key design features of the new holographic interferometer. This system provides uniform exposure of the grating while matraming stable interterence fringes.
Before being split into two separate beam paths, the laser boam is raster scanned in two dimensions using the displacement introduced by rotation of a parallel plate. "The plate of glass is mounted within a dual-axis rotation mount. The scanner includes a computer-controlled stepper motor geared to have the necessary angular resolution while producing linear scan dimensions of up to $11-15 \mathrm{~mm}$. After exiting the laser scamner, the beam is transported to a second table supporting the interferometer, is split into two equal infensity beams, and is directed toward two separate telescopes. The beam-splitter configuration is a critical factor in maintaining stable interference fringes at the recording plane. while simulaneously scaming the laser heam over the full aperture of the two arms of the interferometer.

The primary requirement to achieve a stable interferometer configuration involves splitting the light into (wo arms that gain equal amounts of increased path length when the beam is subjected to angular deviation prior o the split. This requirement is fulfilled boh in sign and magnitude by using the following design criteria. First, the interferometer must maintain left-to-right sense between the two beamlines. This is achieved by selling the difference in the number of mirrors between the two beamlines to be an even number. Second ace inating plate, with the same thickness as the heam splitter, must be placed within the transmitted beam at the same angle of incidence that exists for the beam splitter. The beam that reflects from the back surfice of the bean splitter acquires the same optical path difference as the beam that transmits through both the beam splitter and the compensating plate.

This is the same criteriathat wasestablished for the Micheison interferoneter? which used an incoherene white light souree. The principles of the Michelsoid interferometer are now being extended to coherent laser light to achieve stable interterence fringes during lwo dimensional scamning of the laser beam. This 
design principle provides greatly reduced sensitivity to both laser mispointing during exposure, and angular rotation of the laser beam during scanning. In addition, parallel beam displacement is used as the beam-scanning technique. rather than angle scanning. to eliminate virtually all sensitivity to pre-splitter beam motion.

The two upcollimating telescopes, one within each arm of the interferomeler, consist of an input objective, a spatial filter assembly, and an output collimating lens. Refractive telescopes are chosen to minimize sensitivity to vibration and reduce wavefront error. All optical elements--lenses, mirrors, heam splitters, and recording haterials--are rigidly mounted to reduce vibration effects. In addition. the table for the interferometer is isolated from building vibrations by four pressurized support legs. To further isolate the system from vibration sources, thermal sources, and turbulence, it is located in a "room within a room" environment. In practice, it is found that the holographic system performs best during late night hours when most perturbation sources are reduced or absent.

\section{Grating Fabrication}

The fabrication of holographic diffraction gratings involves a combination of photolithographic and interferometric processes involving a variety of optical and chemical techniques. Highlights of the fabrication process are outlined in the flowchart shown in Table 50.I. The design of a holographic grating involves the determination of proper groove profile (width, height, and shape) and the selection of optical materials used for the substrate (anti-reflection coatings and surface-relief layer). Two activities are performed after the grating is designed. Substrate preparation includes application of three thin-film coatings: an antireflection coating, a primer, and a photosensitive resist layer. This is followed by a baking of the substrate to remove the majority of the resist solvent. Optical inspection of the surfaces and high-resolution wavefront analysis of the optic complete the substrate preparation procedure.

In parallel with this activity, the interferometer is configured according to the grating size and groove width required. First, the laser source is aligned to operate at the necessary power output with a single longitudinal mode and single spatial mode. Second, the beam transport, scanner, and telescope optics are aligned. Third, laser scanning ento a photodetector is performed to determine the effective time-averaged fluence at the recording plane.

Once this calibration is complete, a non-interferometric exposure is applied 10 a resist-coated sample to quantify the relationship between exposure and material removal. These results determine both the bias exposure and the interferometric exposure needed to produce the required surface-relief profile. Exposed photoresist is developed in a sodium hydroxide and water mixture according to predetermined relationships between the baking time, the exposure, and the developer concentration. Once the grating has been chemically developed, gra ding mounting, efficiency measurements, and wavef ront testing are performed as the final steps of the procedure. 
Table 50.1: The fabrication of holographic diffaction gratings involves at combination of photolithographic and interferorhetric processes utilizing a variety of optical and chemical techniques.

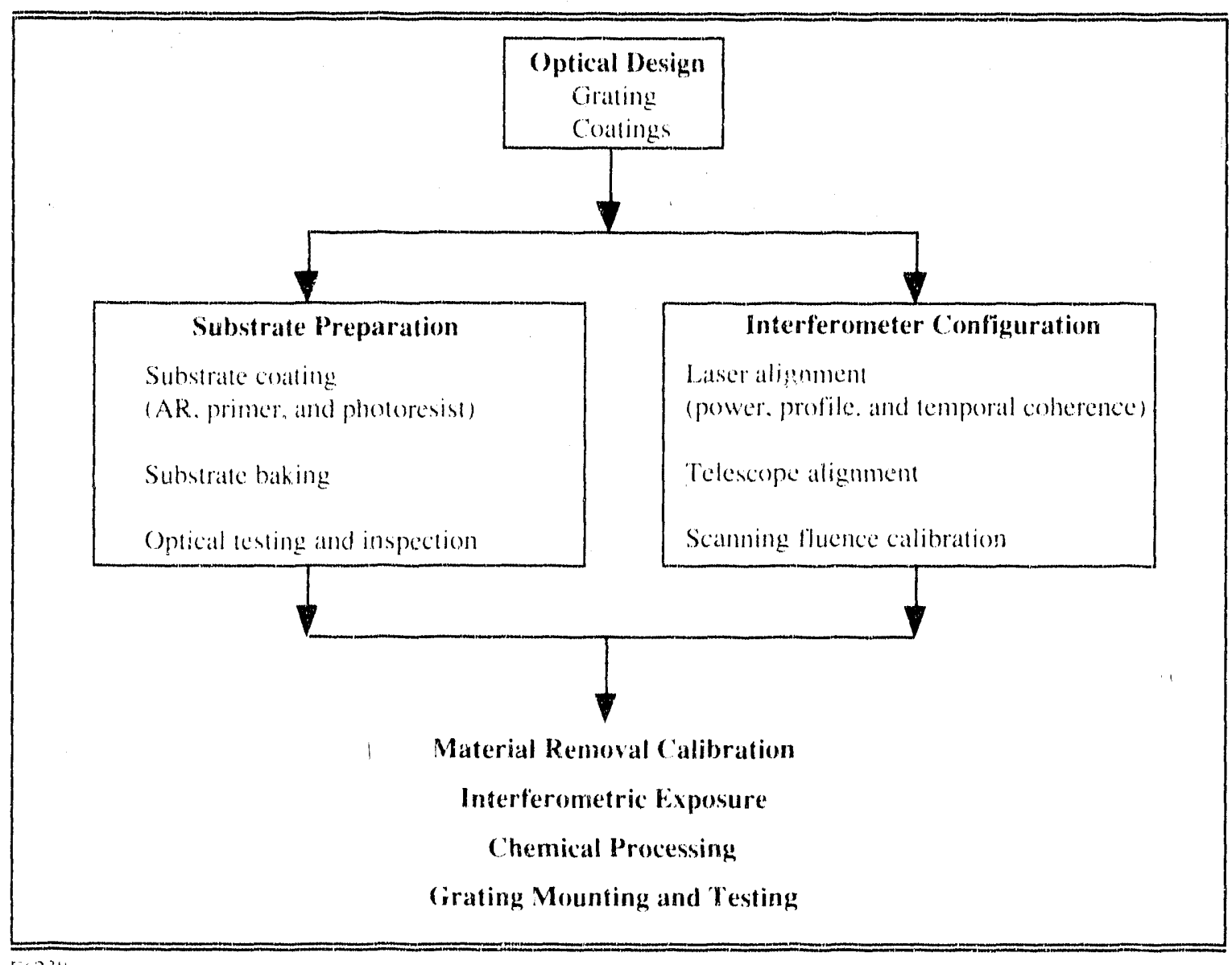

The scanning electron microscope (SEM) is used to characterize the surface relicf of the holographic grating that exhibits unity diffraction efficiency. The size and shape of the grooves are shown in Fig. 50.4. Close-up examination of the grooves shows that the profile is nonsinusoidal. A measured diftactionefficiency verses incident-angle curve, shown in Fig. 50 5, indicates greater angular sensitivity than that predicted for the optimum sinusoidal groove shape. In addition. it has been found that grating efficiency is less sensitive to mean exposure than is theoretically predicted. This relative insensitivity to mean exposure. the observed increase in angular sensitivity, and the SEM determin. ation of nonsinusoidal groove shope can potentially be explained by ultrahigh visibility logether with a malerial- moval saturation effect. Athough this effect needs to be characterized further for applications in grating replication. grating of unity efficiency have immediate applications prior to obtaining at complete understanding. 
Fig. 50.4

Close-up examination of a holographic diffraction grating, using the scanning electron microscope (SEM), shows that the surface relief of a high-efficiency grating is nonsinusoidal.

Fig. 50.5

$10(0 \%$ intrinsic diffaction efficiency has been demonstrated with nonsinusoidal surface relief. Theoretical modeling predicts the diffraction eficiency of a holographic grating to possess a weak dependence on the incident angle as shown by the gradual artation in the solid curve. Experimental meatsurements of the actual surfacerelief profile show a greater angular sensitivity.
ADVANCED TECHNOLOGY DEVELOPMENTS

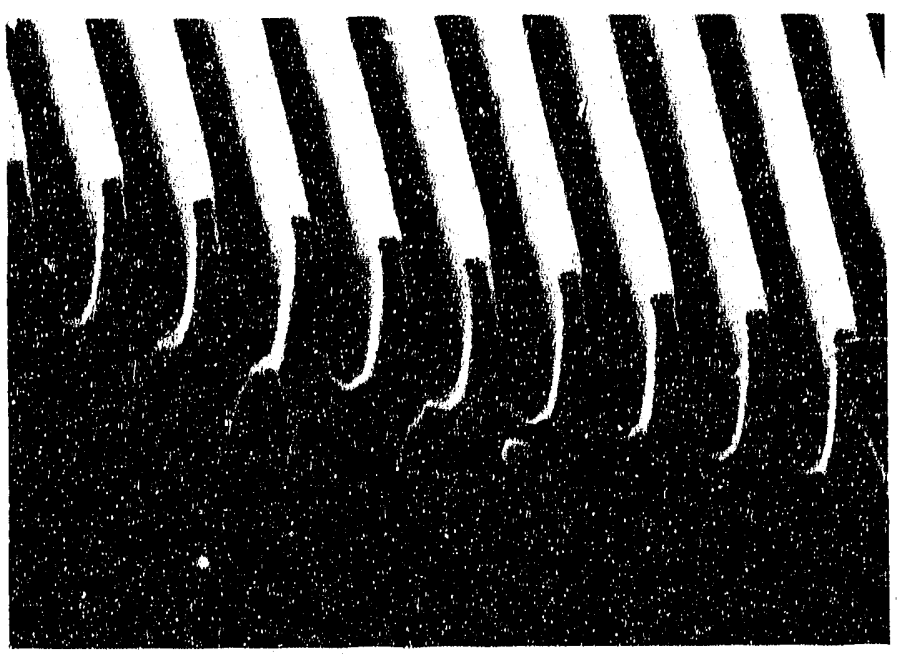

E6140

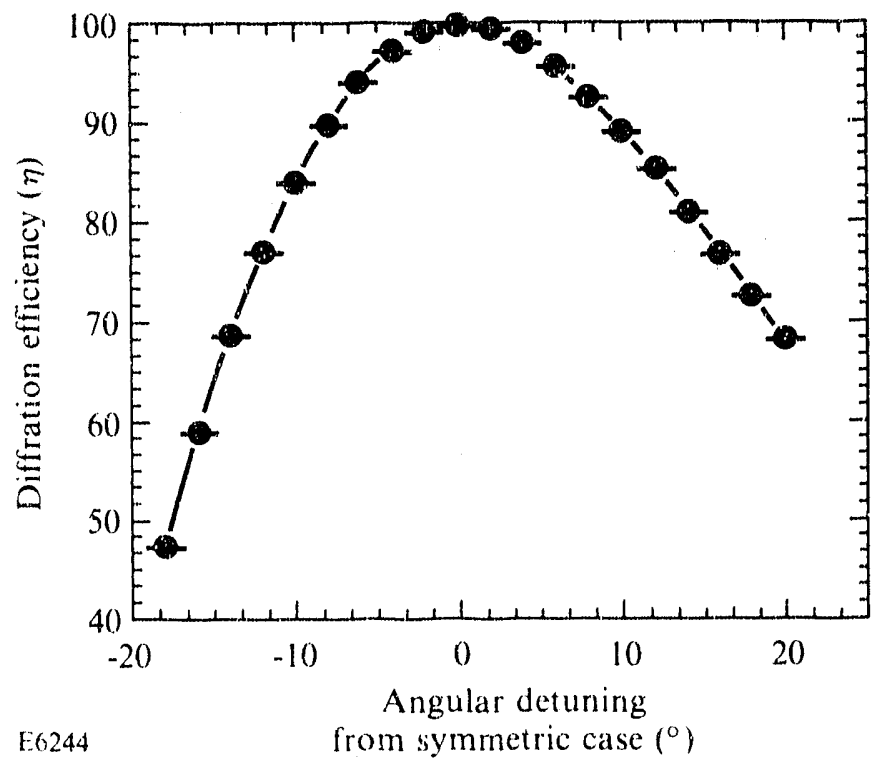

\section{Laser Applications}

A wide variely of applications exist for high-efficiency, high-damagethreshold, holographic diffraction gratings. Currently, a set of gratings designed for infrared $(\lambda=1054 \mathrm{~nm}$ ) laser light is used in the driver line of the OMEGA system to provide the angular dispersion required for laser-beam smoothing, ${ }^{8}$ Several sets of holographic gratings, with a greater amount of angular dispersion, will be required for the driver lines of the OMEGA Upgrade laser. In addition, as schematically illustrated in Fig. 50.6, broadband frequency conversion can be accomplished with gratings placed on either side of the frequency-conversion crystals. Spectrally resolved phase matching within the tripler is achieved with 
Fig. 50.6

Broadhand frequency tripling and phase

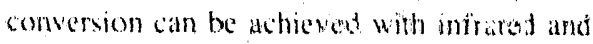
whaviolet diffration gratings. 'They are phaced on either sde of the KDP erystals used for frequency conversion of the high-power. infrated laser light

Fig. 50.7

Highly dispersive transmissongratings placed within a regenerative amplitier. are used to increase the spescral purity of the light inside the later cavity. A 5()-p pulse is stretched to over 5 ns as a first demonseration of this use of holographic gratings. the infrared grating, while the desired amount of color cycling is obtained by protially cancelling the angular dispersion caused by the infrared grating with the .rigular dispersion of an ultraviolet grating.

Other applications for infrared gratings include pulse expansion, pulse compression. and pulse shaping of pulsed laser beams. In each of these schemes, highly dispersive transmission gratings provide several design advantages. They offer greater dynamic range. compactness, high damage threshold, and high diffraction efficiency. As shown in Fig. 50.7. highly dispersive gratings, placed within a regenerative amplifier, can he used to increase spectral purity of the light inside the laser cavity. The pulse width of a Fourier-transform-limitedlaser pulse in increased as its bandwidth is decreased. Todemonstrate this use of holographic gratings. a 50-ps pulse has ban streched to over 5 ns by using a grating with $2(0) \mu$ rad per angstrom angular dispersion. Pulace expansion to 10 ns is possible using the configuration in Fig. 50.7. As another example of the rulse-width control offered by transmission gratings. Fig. 50.8 shows a scheme for pulse compression accomplished using four high-damage-threshold holographic gratingm. A rigidly mounted thin substrate is used for the fourth grating to minimize nonlineat beam effects. Altemate strategies of reducing the amount of nomlinear phase are being investigated.

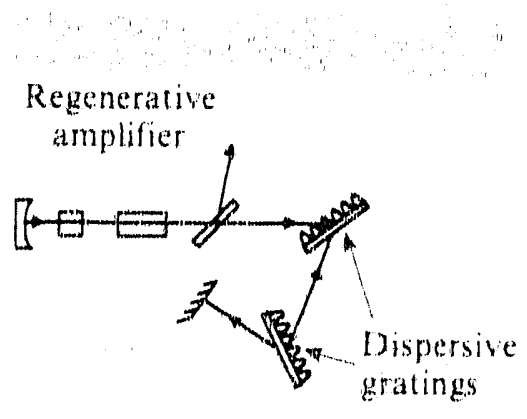

E(2)11

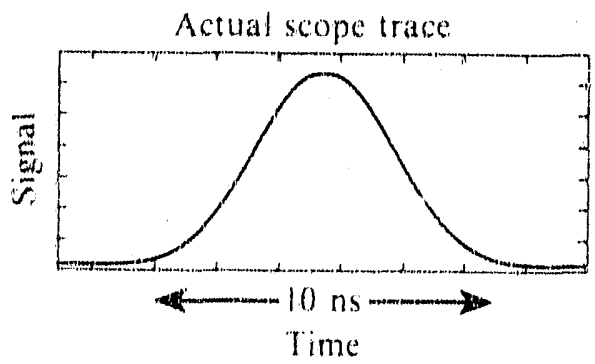




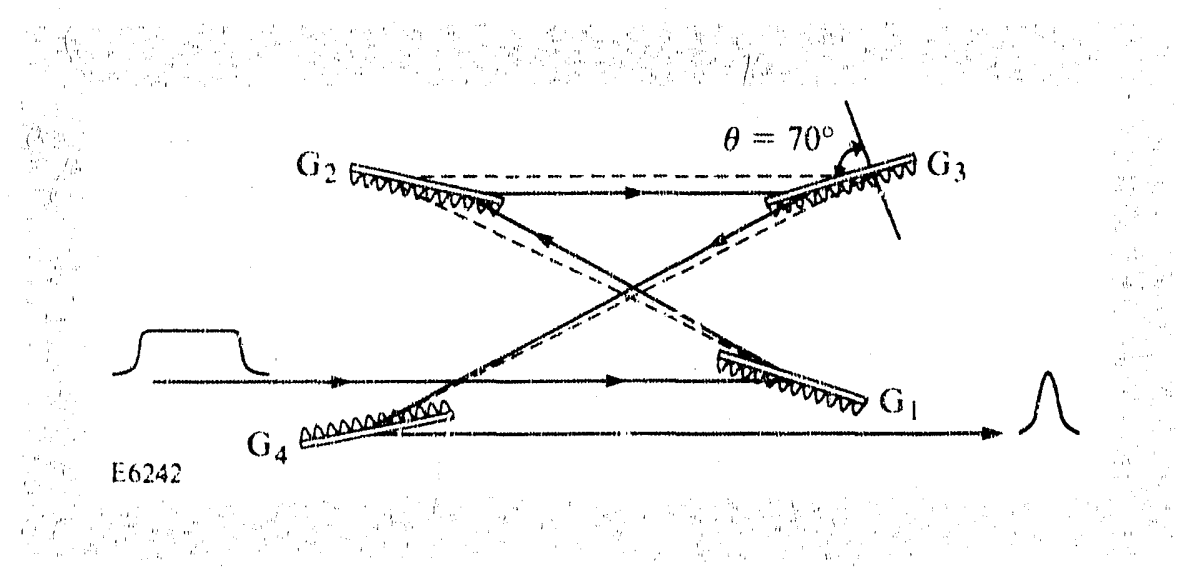

Fig. 50.8

Compression of a laser pulse with a duration of hundreds (f picoseconds to a much shorter duration of a few picoseconds can be accomplished with four high-damage-threshold holographic graings.
A novel method of pulse shaping (see Fig. 50.9), involving a mapping from time to space, followed by a spatial-filtering operation, and then a reverse mapping from space to time, is made more practicable using transmission gratings. An appropriately designed spatial filter located at the focal plane of the grating spectrometer gives rise 10 a pulse shape at the output of the second grating pair that is similar to the transmittance function of the filter. This pulseshaping scheme is currently under development for use on the OMEC 4 Upgrade laser system.

Additional applications of high-power, high-dispersion transmission gratings involve its function as a spectral filter. These gratings provide excelient blocking capability at the Fourier plane for measurement of small-signal gain, for ASE suppression in amplifier subsystems, and many other si' .ations, many of which remain to be demonstrated. Furthermore, the transmission gataing, based on diffraction from a surface relief, provides several advantages for the design of spectroscopic instruments. Compact optical configurations capable of high angular dispersion and efficient over a broad wavelength range are possible. A highly dispersive and low-noise spectrometer is illustrated in Fig. 50.10.

\section{Conclusion}

High-efficiency holographic gratings, exhibiting unity diffaction efficiency, have been demonstrated for use in high-power laser systems. A novel interlerometric technique, which incorporates pars.'lel beam scanning and a pointing-in.:- risitive optical configuration, is used to obtain uniform irradiation of the recorditiz medium while maintaining stable interference fringes. Largeaperture gratings, covering a wide range of groove spacings, can be manufactured with this interferometric exposure technique. Numerous applications exist for these high-efficiency and high-damage-threshold holographic gratings. Laserbeam snoothing and pulse-shaping techniques, many of which have been invented and developed at LLE, incorporate these gratings at physical apertures ranging between several centimeters to tens of centimeters. In addition, highly dispersive holographic diffraction gratings are a central component to pulseexpansion and pulse-compression schemes

Furthermore, these new gratings offer the possibility of building highresolution and high-signal-to-noise spectroscopic instruments. Future research 


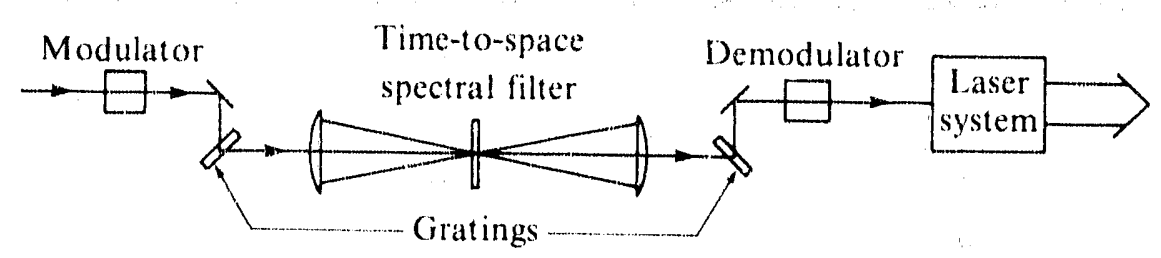

Example: Foot-pulse evolution

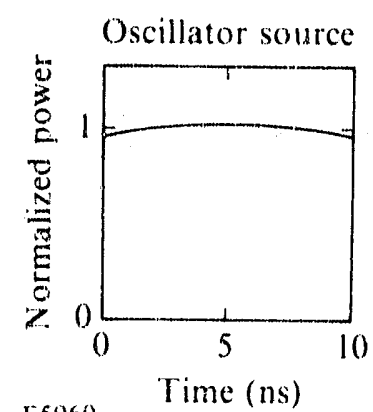

E5969

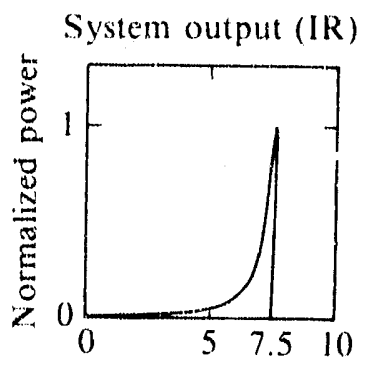

Time (ns)

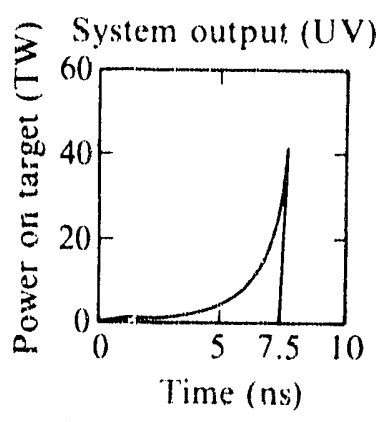

Fig. $5(0.30$

The transmission grating provides several advantages for the design of spectroscopic instruments. Compact configurations capable of high angular dispersion and efficient over a broad witelength range are possible.
Fig. 50.9

Spectral pulse shajing involves a mapping from time to space, followed by a spatialfillering operation, and then a reverse mapping from space to time. Placement of an apodizer at the focal plane of the grating spectrometer gives rise to a pulse shape, at the output of the second grating pair, similar to the transmittance function of the filter.

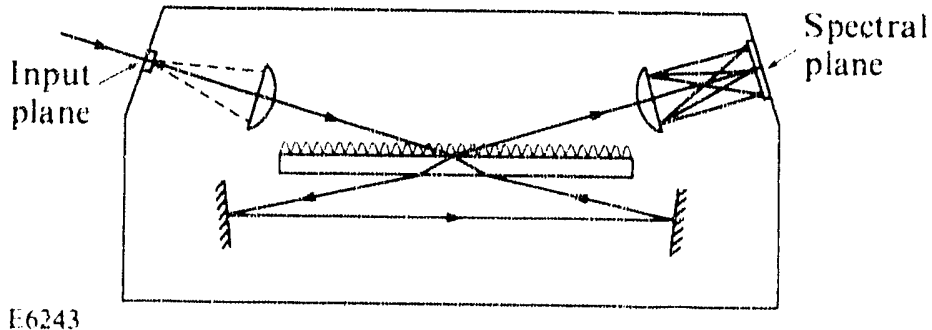

is aimed at the development of replication techniques necessary for the production of large-aperture uitraviolet gratings that would be used for broadband frequency conversion and phase conversion of laser light on the OMEGA Upgrade laser system.

\section{ACKNOWLEDGIMENT}

Thin work wats supported by the U.S. Departmen of Energy Office of Inertial confinement Fusion under agreerrent No. DE.FC 13-85DP4012(K) and by the Laser Fusion Feasibility Project at the L atboratory for Laser Eincrgetics, whe h is sponsored by the New York State Energy Research and Development Authority and the University of Rochester. 


\section{REFERENCES}

1. R. Collier, C. Burckhardt, and L. Lin, Optical Holography (Academic Press, New York, 1971).

2. Electromagnetic Theory of Gratings, edited by R. Petit (Springer-Verlag, Berlin, 1980).

3. 'T. K. Gaylord and M. G. Moharam, Proc. IEEE 73, 894 (1985).

4. D. Maystre, J. Opt. Soc. Am. 68, 490 (1978).

5. L. Li, University of Arizona, and Y. Lin, University of Rochester, private communications.

6. M. Miler, Sov. J. Opt. Technol. 48, 491 (1981).

7. A. A. Michelson, Am. J. Sci. 22, 120 (1881).

8. LLE Review 37, 40 (1988).

\section{B Shaping and Measuring a Laser Pulse Using Spectral Beam Deflection}

The capability to produce laser pulses of arbitrary temporal shape is important in areas of opticai communication, atomic and molecular spectroscopy, ${ }^{2}$ and laser fusion. ${ }^{3}$ Previous pulse-shaping techniques have achieved moderate success in these areas. ${ }^{4-6}$ The new approach discussed here, using the technique of spectral beam deflection (SBD), offers the potential of higher temporal resolution combined with the flexibility of beam-size variation and aperture-position variation during the pulse. SBD can produce the high-precision multinanosecond pulses required for laser fusion applications, and it is compatible with standard pulse-compression techniques for producing shaped subpicosecond pulses. Although SBD is applied here mainly to pulse shaping and beam-size shaping, it is a general technique that can be used for any application that requires laserbeam scanning for illuminating an object or for reading and writing informazion.

Laser-beam temporal pulse shaping has previously been yerformed in both the time domain ${ }^{4.5}$ and frequency domain. ${ }^{6}$ Time-domain shaping [Fig. 50.11 (a)] is accomplished by passing the beam through, for instance, a Pockels cell placed between two polarizers that will cause time-dependent attenuation of the beam when driven by a shaped electrical pulse. This approach relies on electronic techniques for constructing the required temporal electrical shape. At present, it has been difficult to produce very complicated or very fast rising pulses. The second approach, pulse shaping in the frequency domain [(Fig. 50.11(b)], is accomplished by spatially separating the spectral components of the beam (using a diffraction grating) and modifying the amplitude and/or phase of these components to produce the spectrum that corresponds to the desired temporal pulse shape. When the spectral components are recombined, very complicated 
(a) Time domain

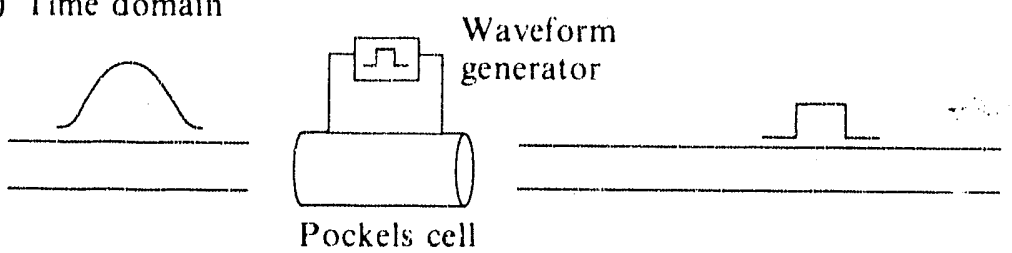

(b) Frequency domain

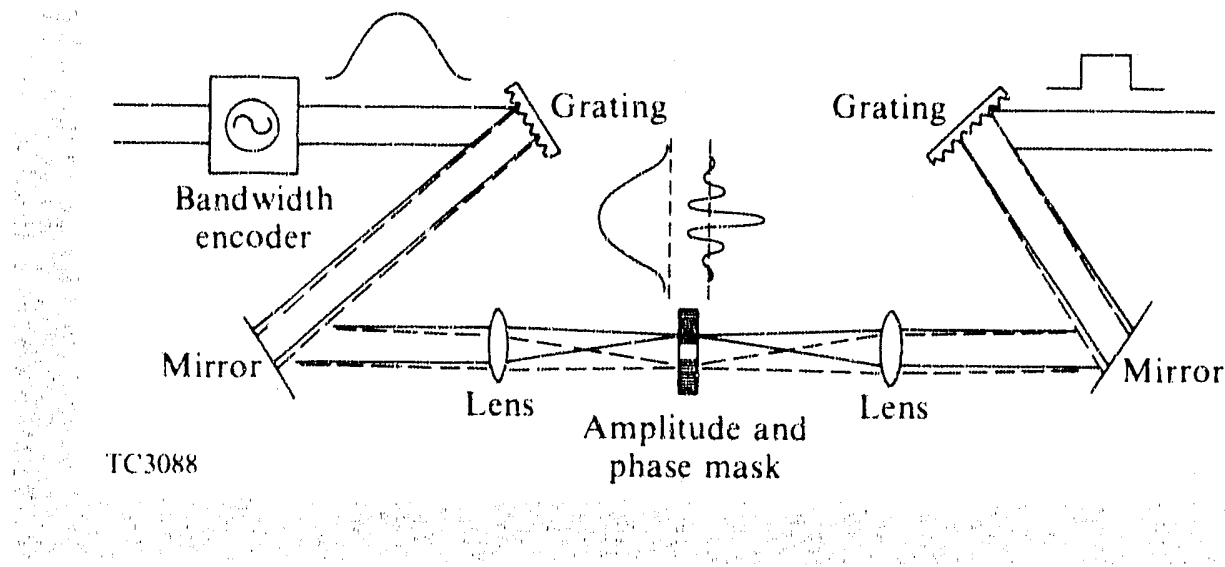

Fig. 50.11

These are two generic techniques for laser-beam f.lse shaping: (a) time-domain shaping uses a temporally shaped electrical current to produce a time-varying attenuation of the beam; (b) frequency-domain shaping separates the pulse into its spectral components, and then modifies the relative amplutudes and phases of these components to produce the spectral distribution of the desired pulse shape. pulse shapes can, in principle, be produced. However, in order for the spectral components to combine properly, generally both the amplitudes and phases must be fashioned 10 an extremely high level of precision, since each spectral component will affect the pulse throughout its entire temporal duration. Such precision has been difficult to ottain, and as a result, this technique has been applied only to relatively simple pulse shapes.

The pulse-shaping technique discussed here is a hybrid between the timedomain and frequency-domain approaches, and it uses the best features of each. It has the advantage of the time-domain approach in that each temporal region of the pulse is constructed relatively independently of the other regions. However, unlike time-domain shaping the pulse is not constructed using time-varying electrical pulses but rather using static filters, which is one of the attractive features of frequency-domain shaping. This hybrid approach is accomplished by passing the beam through a device that causes temporal deflection of the light (Fig. 50.12). When the beam is focused by a lens, the position of the focal point will change in time as the beam is deflected, creating a one-to-one mapping between time during the pulse and spatial position in the focal plane. Intensity attenuation in a spatial region in the focal plane will then produce attenuation in the corresponding temporal region of the pulse when the beam is returned to its far-field position.

Pulse shaping by beam deflection has been previously examined using electro-optic prisms to produce the deflection. ${ }^{7}$ Here we use SBD to produce the beam deflection. This results in greater flexibility and potentially higher resolution. For example, SBD is sufficiently flexible that it can directly accommodate the option of pulse compression to produce shaped subpicosecond pulses. Also, with 


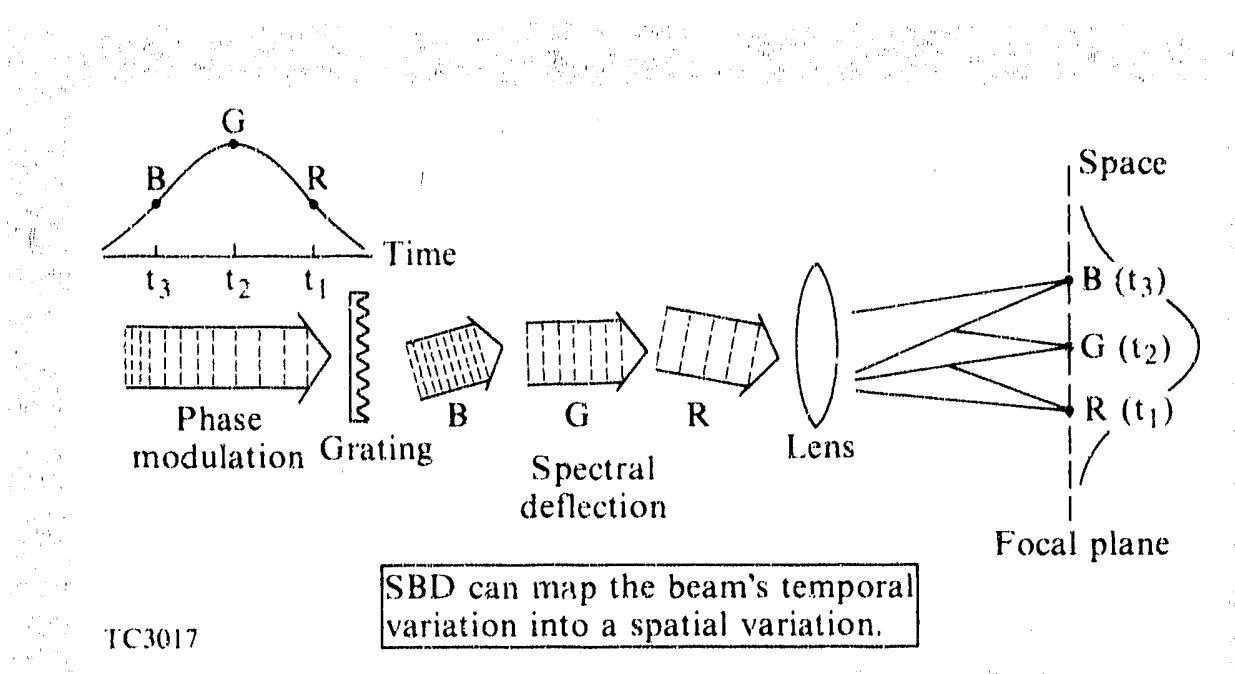

Fig. 50.12

Spectral beam deflection (SBD) maps a beam's temporal variation into a spatial variation by means of whole-beandeflection. The deflection is produced by coarse dispersion of a frequency chirp that was imposed upon the beam. a small extension of the pulse-shaping configuration, SBD will allow temporal changes of the beam size as well as changes of the beam location within the aperture (including the production of multiple bearns within the same aperture). Finally, the time-10-space mapping produced by beam deflection has been proposed for constructing ari sptical streak camera to measure the temporal pulse shape of a laser beam. ${ }^{8.9}$ A more general configuration, based on SBD, is discussed here.

\section{Description of Spectral Beam Deflection}

The method for achieving beam deflection with the SBD pulse-shaping technique is illustrated in Fig. 50.12. Starting with a generic pulse, the first step is to impose phase-modulated bandwidth upon the beam such that the instantaneous frequency (time derivative of the phase) is changing monotonically throughout the pulse. This assigns to each point in time during the pulse a unique characteristic spectral frequency. The required modulation bandwidth is typically 10-100 times the Fourier-limited bandwidth of the desired pulse shape. (The higher the bandwidth, the greater the possible temporal resolution). Two methods of bandwidth generation are currently under investigation. One approach is to pass the beam through an electro-optic (E-O) modulator driven by microwaves. The modulation frequency and phase are chosen so that a half cycle, containing the extremes of the bandwidth, encompasses the pulse, with the central frequency at (or near) li. eak of the pulse. After the pulse shaping, excess bandwidth can in principle be removed by passing the beam through a modulator a second time, but $180^{\circ}$ out of phase. The second approach being considered for bandwidth generation is to chirp the pulse by passing it through an optical fiber. This approach has been used extensively for pulse compression to generate subpicosecond laser pulses. It has the advantage of centering the frequency chirp around the peak of the pulse without requiring the synchronization needed for an E-O modulator. The disadvantage is that the bandwidth cannot be removed (except by using a properly synchronized E-O modulator), and also the early and late parts of the pulse might not be properly chirped and could require some truncation. 
Deflection of the beam is now achieved using a diffraction grating. Each point in the pulse will be deflected in a direction determined by a small spectral range around the instantaneous frequency of the phase-modulated bandwidth. When the beam is focused, the deflection will cause each region of the pulse to be mapped into a unique region in the focal plane (Fig. 50.12). (This technique was applied in Ref. 9 for application to an optical streak camera.) There will be some spatial overlap of neighboring temporal regions because of the finite spot size of the beam, the beam's intrinsic bandwidth, and because of spectral components that are common to both temporal regions. The amount of overlap determines the resolution of this technique.

Spectral separation of the bandwidth in the focal plane of a lens is very suggestive of the frequency-domain pulse-shaping approach |Fig. 50.11(b)|. However, there is one important difference. Frequency-domain shaping requires high spectral resolution, and it has been applied to situations where the pulse is ban Jwidth limiled (i.e., the spectrum is dominated by the amplitude variation of the beam). In that situation there is no direct relationship between a frequency interval and a temporal range in the pulse, and both the phase and amplitude must be shaped very precisely in order for the spectral components to recombine into the required pulse shape. (Specifically, it is the Fourier transform of the pulse that must be shaped.) For SBD, the bandwidth is chosen sufficiently large that the spectrum is dominated by the phase modulation, and an "instantaneous" frequency relating time and space is well determined. In the focal plane there is a sufficiently large spectral overlap that a close correspondence between time and instantaneous frequency can be maintained. The result of the spectral dispersion with SBD is predominantly whole-beam deflection. Because of the direct spacetime mapping, the pulse shape itself is constructed and not its Fourier transform. Only amplitude attenuation, and not phase modification, is required. The E-O modulator and grating combination used for SBD is similar to the configuration used for the beam-smoothing technique known as smoothing by spectral dispersion (SSD). ${ }^{10}$ Indeed, in some SSD configurations, whole-beam deflection has been observed.

An alternate method for producing temporal beam deflection is to use an electro-optic prism. ${ }^{7}$ The advantage of the technique proposed here is that the phase-modulated bandwidth and the beam deflection are produced by two separate components. This results in considerably more flexibility, and it allows each component to be separately optimized to maximize its effect. With the E-O prism, beam defiection must be imposed electro-optically, and it can only be removed by passing the beam a second time through a properly synchronized E-O prism. With SBD, pulse shaping can be performed using totally passive optical components for those applications that do not require removal of the bandwidth, thus avoiding the complications of synchronization with the pulse. The bandwidth can be imposed with an optical fiber, and the deflection is imposed and removed with diffraction gratings. For applications that do require bandwidth removal or if there are advantages for imposing the bandwidth electro-optically rather than with a fiber, then SBD will require the same level of synchronization as the E-O prism. However, there are still advantages to separating the E-O component from the deflection component as done in SBD. 
First, the diffraction gratings used with SBD can generate considerably more angular dispersion than prisms, producing a greater amount of deflection in the focal plane of a lens. Second, multiple-pass configurations can be optimized for each component separately for increasing either the band width or the dispersion or both. Third, the beam size can be chosen optimally for each component: it is easiest to impose $\mathrm{E}-\mathrm{O}$ modulation on a small beam whereas high spectral resolution is obtained with a large beam. Finally, the same bandwidth that is imposed for SBD can also be used for additional modifications of the beam, such as changing the beam size during the pulse, as discussed in the next section.

\section{Applications of SBD}

We consider four applications of spectral beam deflection: (1) pulse shaping; (2) combining pulse shaping with pulse stretching (and compression); (3) changing the beam size and shape during the pulse; and (4) measuring the beam intensity as a function of time (optical streak camera).

\section{Pulse Shaping}

Pulse shaping is accomplished by placing attenuation filters in the focal plane where the beam's temporal variation has been mapped into a spatial variation (Fig. 50.13). The spatial variation of the attenuation is the same as the desired temporal shape, except for a scale adjustment to account for any nonlinearity in the space-time mapping. (For sinusoidal E-O modulation, the space variable $x$ in the focal plane and time variable $t$ are related by $x \sim \sin \omega_{m} t$, where $\omega_{m} / 2 \pi$ is the oscillation frequency of the modulator. Since the modulation frequency is chosen such that there is less than $1 / 2$ of a cycle across the pulse, with the center frequency at the center of the pulse, this mapping is single valued.) The amplitude attenuation can be performed by separate filters for each individual

Fig. 50.13

SBD pulse shaping is a hybrid between timedomain and frequency-domain shaping. Spatial arnplitude attenuation follows the temporal shape of the beatm. (Phase modification is not required.) pulse shape, or by a general two-dimensi nnal addressable array of liquid-crystal polarizer elements that can be modified electronically. Modulators made from liquid-crystal display cells have been used for phase modification. " They can also be used for amplitude attenuation by inducing a polarization rotation followed by a polarizing analyzer.

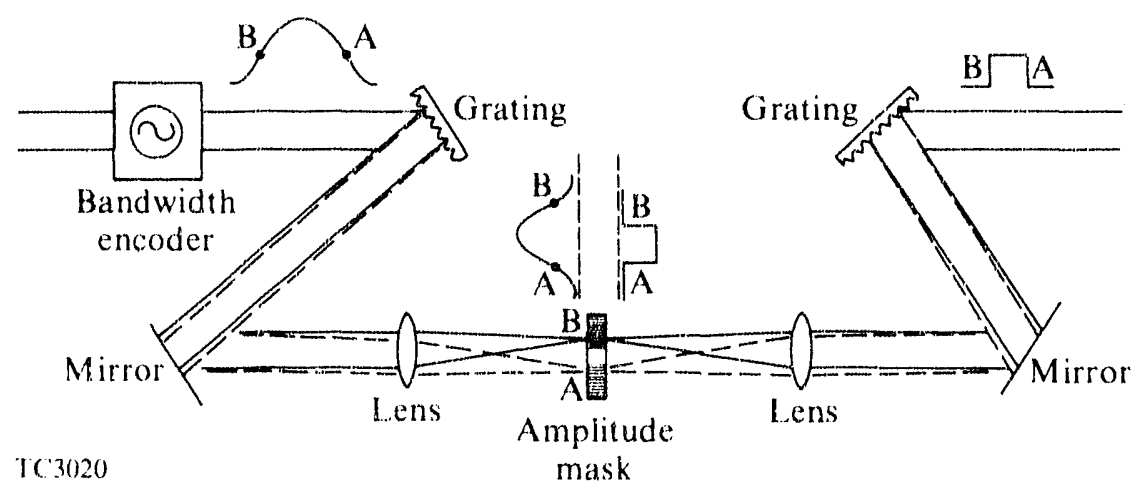


After the spectral encoding and filtration, the beam is recollimated with a lens and the spectral dispersion is removed with a second grating. At this point, temporal pulse shaping has been achieved. In general, on!y a small fraction of the encoded bandwidth has been used to shape the pulse, and the remainder is still in the form of phase modulation. The foliowing options are available for continued manipulation of the beam:

(a) If the excess bandwidth remaining on the beam is too large (as can happen for a frequency-tripled laser that has a narrow bandwidth acceptance for high-efficiency frequency conversion), then a majority of the bandwidth can be removed by passing the beam through an $\mathrm{E}-\mathrm{O}$ modulator a second time operating at the same frequency and modulation index but $180^{\circ}$ out of phase.

(b) The bandwidth can be used for other applications such as beam-size shaping.

2. Combining Pulse Shaping with Pulse Stretching

Pulse shaping by SBD can be further enhanced by combining it with the option of pulse stretching or compressing. This is illustrated schematically in Fig. 50.14. The double-grating technique of changing the pulse width for a chirped pulse has been used extensively in picosecond experiments, ${ }^{12,13}$ but it can be used for multi-nanosecond pulses. This technique can be useful when a variety of different pulse widths are required, and it is not convenient to constantly modify the laser oscillator to generate the different pulses. In this case, the oscillaior would produce a Gaussian pulse with al ways the same width. The pulse would be chirped with the quasi-linear portion of sinusoidal E-O modulation; the direction of the chirp will be chosen according to whether the pulse is to be compressed or stretched.

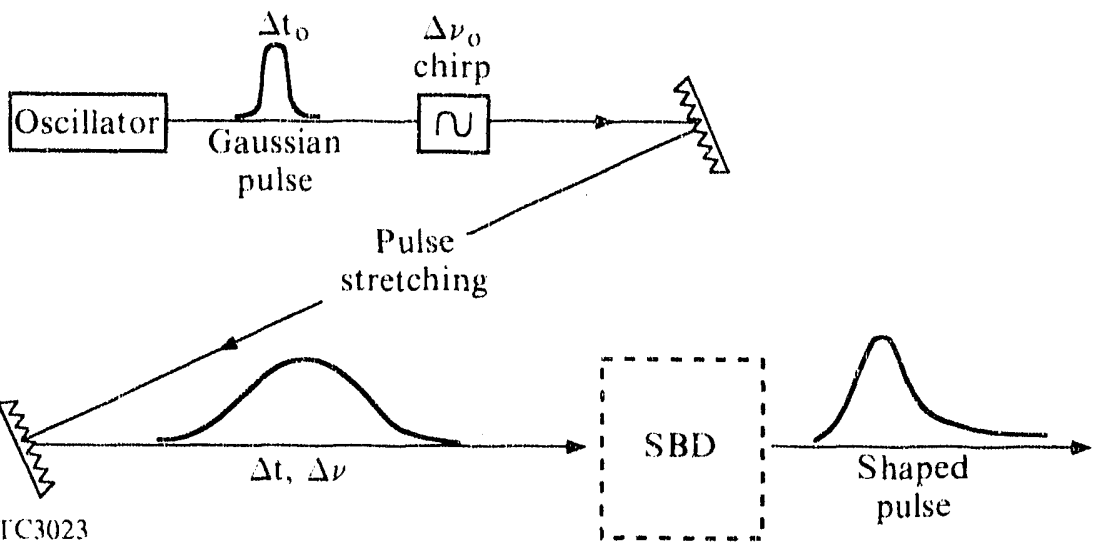

Fig. 50.14

A two-grating configuration can be used to stretch (or compress) the beam prior to SBD shaping. 
Fig. 50.15

The beam size and shape can be varied during the pulse by using cylindrical lenses to focus the beam. This gives access to one dimension of the beam for modification as a function of time.
By passing the beam through the double-grating configuration, the pulse can, in principle, be stretched from say $100 \mathrm{ps}$ to several nanoseconds or compressed to picoseconds. For multi-nanosecond stretching, it may be necessary to pass through the gratings several times to achieve the required time delay. The number of passes is determined by the bandwidth, the grating dispersion, and the distance between gratings. After a large number of passes, it might be necessary to amplify the beam to compensate for grating losses. With a linear chirp, the pulse will maintain a Gaussian temporal profile.

For a proper adjustment of the distance between the gratings, the resulting pulse will remain linearly chirped, though the direction of the chirp can be reversed. This same chirp can now be used with SBD to shape the pulse. No additional bandwidth is required.

Pulse shaping within the double-grating configuration has been previously examined. ${ }^{14,15,16}$ In general, it will provide less temporal resolution than shaping separately by SBD.

\section{Beam-Size Shaping}

The beam size, shape, and position within the aperture can be changed as a function of time by a small variation of the previously mentioned pulse-shaping technique. Instead of using circular lenses to focus the beam, cylindrical lenses are used as illustrated in Fig. 50.15. This allows access in the focal plane to one spatial dimension of the beam as a function of time. By using attenuation filters this dimension can be shaped in time. Returning to the near field the beam is recollimated and the spectral dispersion is removed by a grating. To modify the second dimension of the beam, the beam is passed through a grating oriented to disperse the beam in the perpendicular direction, and the process is repeated. Note that all this manipulation uses the same phase-modulated bandwidth; the bandwidth has to be encoded only once.

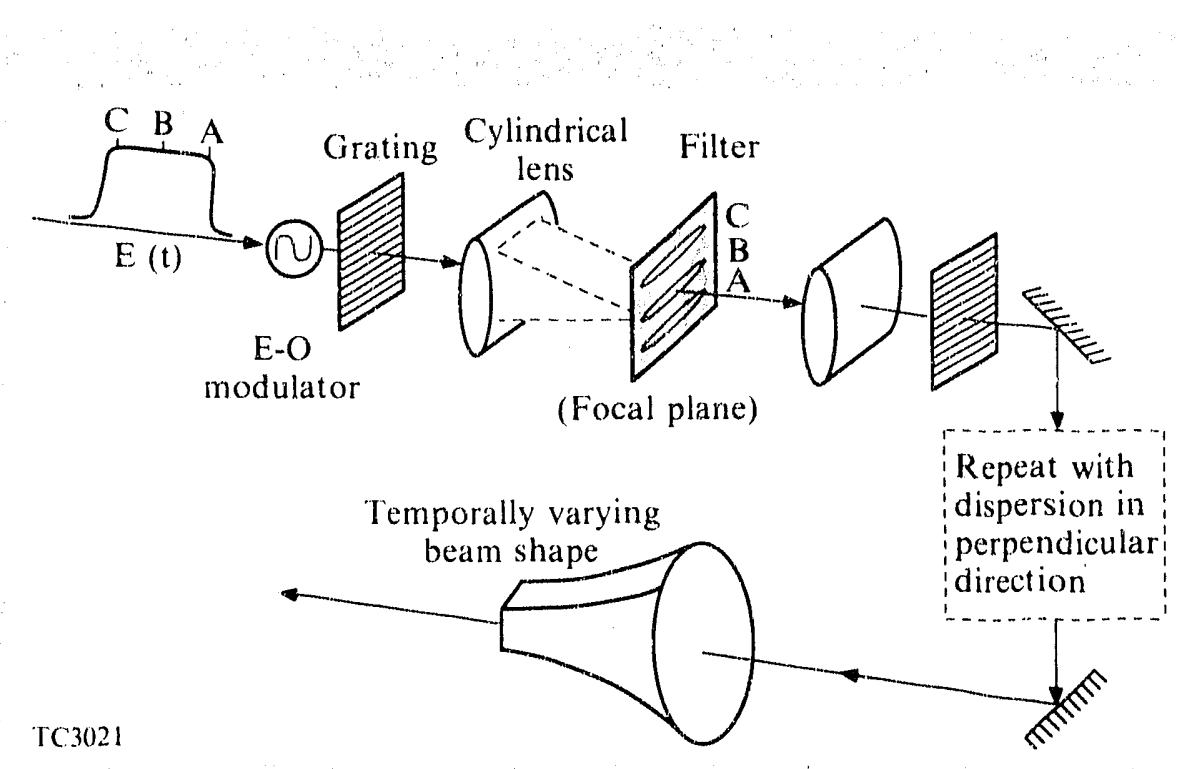




\section{Optical Streak Camera}

The time history of the pulse can be measured by placing a photosensitive device such as film in the focal plane where the beam has been deflected and is spatially displayed. If no other bandwidth has been placed on the beam besides that used for beam deflection and if the intrinsic bandwidth of the pulse is small, then the measuring device can simply be placed in the focal plane of the configuration in Fig. 50.12 (see also Ref. 9). However, if the beam is to be measured after additional bandwidth has been placed on it, such as bandwidth used for beam smoothing, then in general there would no longer be a one-to-one relationship between time during the pulse and spatial position in the focal plane. To accommodate this situation, the config uration in Fig. 50.16 could be used.

The configuration of gratings and E-O modulators shown in Fig. 50.16 accomplishes two goals: (1) any bandwidth on the beam prior to the first grating in the figure will not contribute to the beam deflection and (2) the modulators are not required to be synchronized with the peak of the pulse. It is straightforward to show that the gratings have no effect on any bandwidth that was not imposed by the two modulators. For pre-existing bandwidth the first grating will disperse the bandwidth in the $x$ direction; the second grating will remove that dispersion. Similarly, the third grating will disperse the beam in the $y$ direction, but the fourth grating will remove the dispersion. Only bandwidth imposed by the modulators will be dispersed.

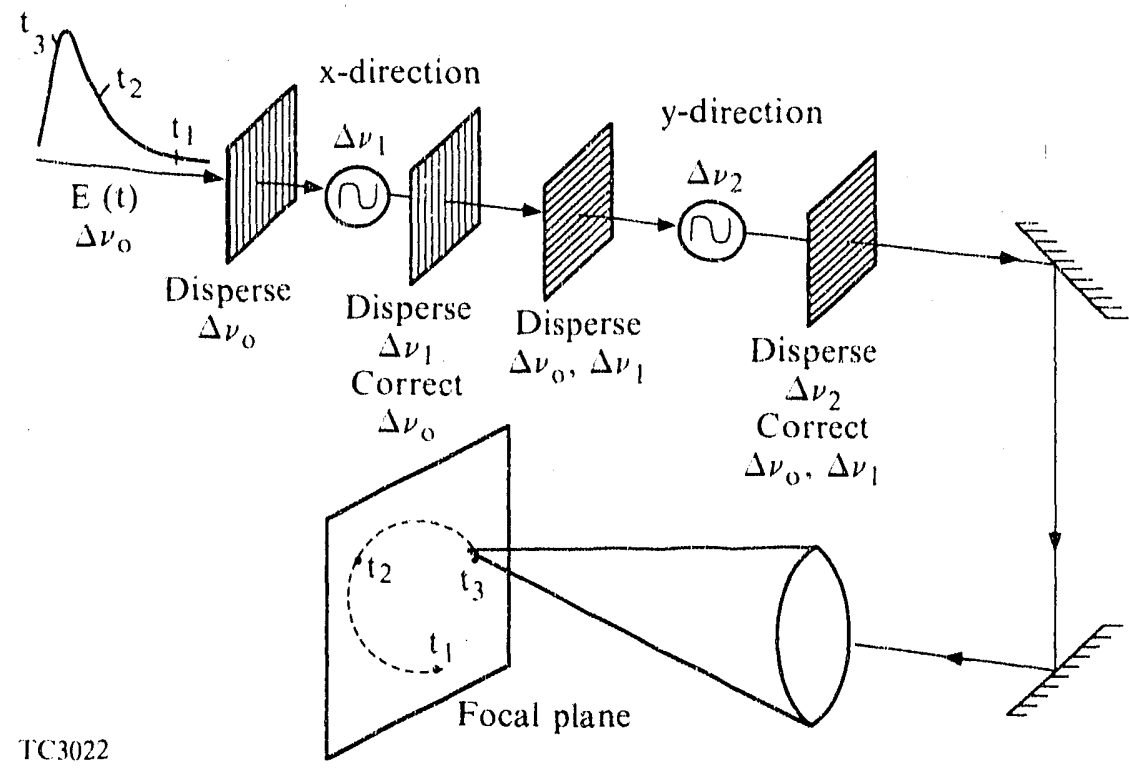

Fig. 50.16

An optical streak camera can be constructed using SBD. The gratings before and after each modulator assure that only the bandwidth imposed by that modulator will be deflected in the specified direction. 
Bandwidth imposed by the first modulator will be dispersed in the $x$ direction by the second grating, and then in the $y$ direction by the third grating. But the $y$-direction dispersion is removed by the fourth grating, leaving this bandwidth dispersed only in the $x$ direction. Bandwidth imposed by the second modulator wit? be dispersed only in the $y$ direction by the fourth grating. Thus, when the beam is focused, it will trace out a pattern that includes deflection in both the $x$ and $y$ directions, determined only by the bandwidth from the two E-O modulators. For sinusoidal modulation, the bearn will be deflected in a circle in the focal plane if the two bandwidths and modulation frequencies are equal, and if the relative phase differs by $90^{\circ}$. In general, any Lissajous pattern can be created.

The relative phase between the bandwidth imposed by the two modulators will vary across the beam because of time delays introduced by the second and third gratings. (This is the same kind of time delay that produces color cycling in SSD.) Thus, different parts of the beam can trace different patterns in the focal plane, and there will not be a unique "streak" for the entire beam. This effect can be small if the grating delay time is small compared to the modulation time of the bandwidth. Otherwise, it will be necessary to pass the beam through an aperture to isolate a portion of the beam for which the phase difference between the bandwidths is relatively constant.

It is not necessary to synchronize the modulators with respect to the peak of the pulse. The only constraint is that the modulation time should be longer than the pulse width so that the pulse will be displayed before the trace repeats itself. The position of the pulse in the focal plane will depend on the phase of the peak of the pulse. but it will not affect measurement of the beam's intensity variation as a function of time.

\section{Examples of Pulse Shaping by SBD}

Pulse shaping by SBD was modeled numerically. The bandwidth was cecomposed into its spectral components. The spot size in the focal plane was taken into consideration when calculating the attenuation through the filter in the focal plane. The calculation includes the effects on shaping resolution based on spatial overlap of different spectral components and the effects of finite focalspot size. The attenuated spectral distribution was recombined to determine the resulting waveform.

In the following examples, we consider the case where the focal-spot size was about $1 / 50$ of the size of the beam-deflection region in the focal plane. We then find the amount of bandwidh required to achieve different pulse shapes.

The first example, Fig. 50.17, shows an attempt to numerically construct a high-order, 1-ns superGaussian pulse (the dashed line) using SBD. Figure 50.17 (a) shows the effect of using only a small amount of bandwidth, $0.5 \AA / \mathrm{ns}$. The shaped puise is fairly well contained in the 1-ns region, but interference between different spectral components has produced intensity oscillations. As the bandwidth is increased in Figs. 50.17(b) and 50.17(c), the superGaussian shape is more clearly reproduced. The increased bandwidth makes the mapping between time and space more accurate so that in the focal plane there ir less spatial overlap between different temporal regions. 


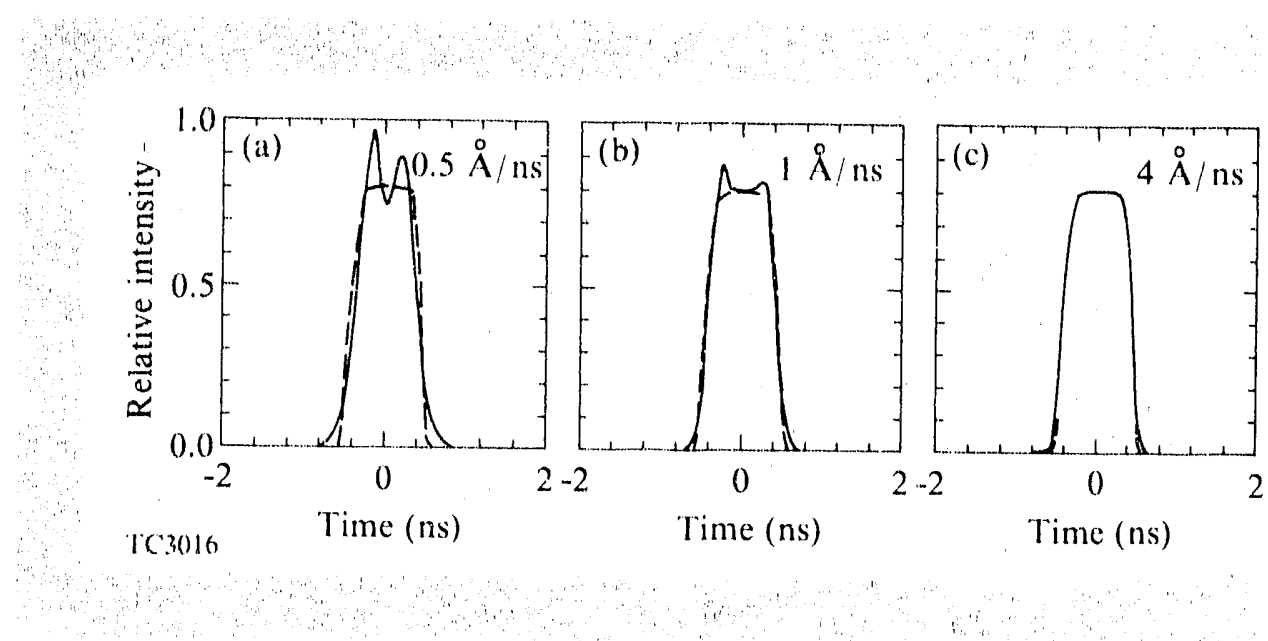

Fig. 5() .17

A numeris...' simulation of SBD (solid line) to construct a high-order, I-ns superGaussian pulse (dashed line). For low bandwidth (a) the pulse is well localized, but the time-space mapping is not sufficiently acc urate to prevent interference between spectral ceruponents that producs intensity modulations. For higher handwidth (c) greater temporal resolution is obtained. A laser wavelengti of $1 \mu \mathrm{m}$ was used, and the beamdeflection length in the focal plane is 50 times larger than the focal-spot diameter.
When the pulse shape is more gently rising, adequate resolution is sbtained with less bandwidth. This is illustrated in Fig. 5().18 for a 7 -ns pulse that could be used for laser-fusion experiments. Here only $2 \AA$ is required. In the other extreme a more steeply rising pulse requires a higher bandwidth. Figure 50.19 shows the result of constructing a 250)-ps superGaussian pulse. A bandwidth of $20 \AA$ was required to produce a rise time of $-1.5 \mathrm{ps}$ per decade, over four decades.

The effect of removing the bandwidth was also modeled by adding E-O bandwidth $180^{\circ}$ out of phase. In all cases it was possible to eliminate or greatly reduce all excess bandwidth.

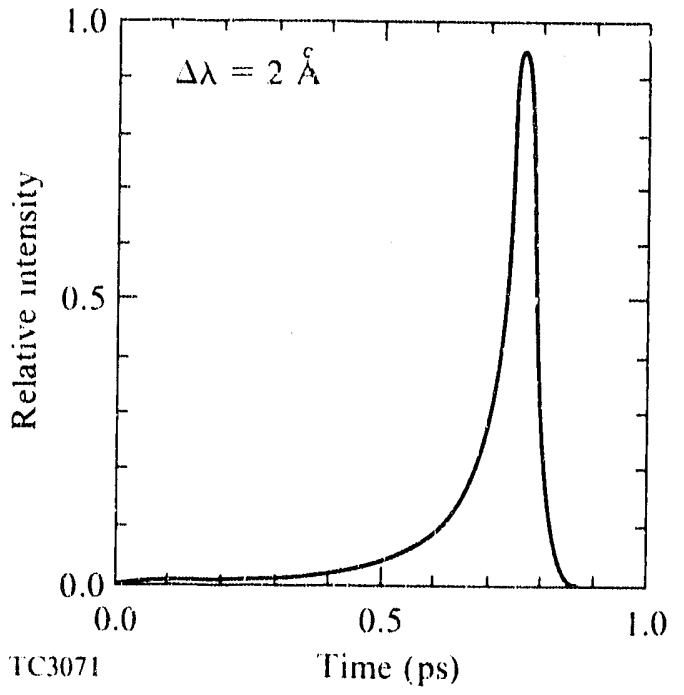

Fig. 50.18

For more gently rising pulses, less bandwidth is needed compared to Fig. 50.17 . 
Fig. 50.19

For faster rising pulses than in Fig. 50.17 , larger bandwidth is required. A bandwidth of $\sim 20 \AA$ was required to produce a rise time of $1.5 \mathrm{ps} /$ decade over a range of four decades.

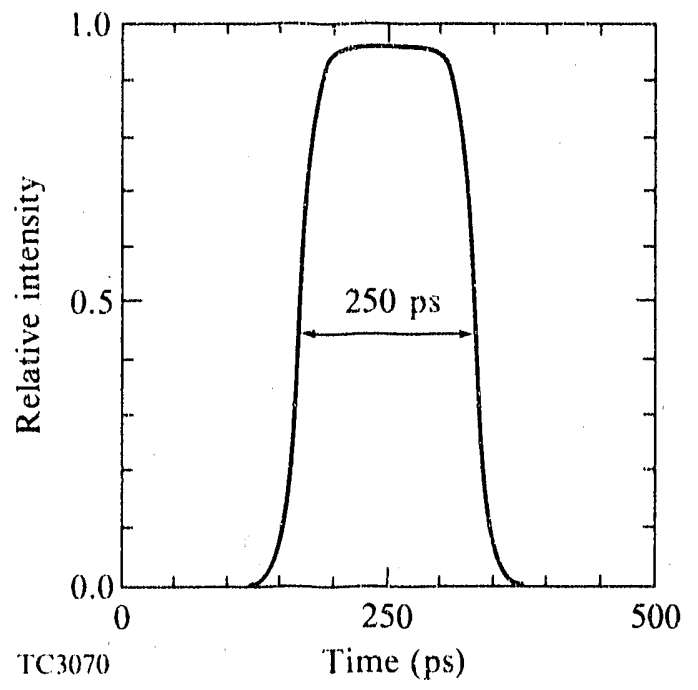

\section{Summary}

Spectral beam deflection (SBD) is a very flexible technique for producing whole-beam deflection. This technique can provide a high-resolution mapping between time during the pulse and spatial position in the focal plane of a lens. Operating in the focal plane, we have the opportunity to modify the beam's temporal characteristics, using static amplitude filters, or to measure the beam's time history.

Four applications of SBD have been examined: (1) pulse shaping, (2) combining beam stretching with pulse shaping, (3) beam-size shaping, and (4) a design for an optical streak camera. Pulse shaping was modeled numerically to determine the effects on resolution from spectral overlap of different temporal regions and from spatial overlap caused by the finite spot size of the beam in the focal plane.

These techniques could be applied to several different areas. Spectral beam deflection can be used wherever laser scanning is required for illuminating objects and for reading and writing information very rapidly. Beam-size shaping might be used in machining or surge $y$ for precision illumination of different portions of an object for different amounts of time. Precision pulse shaping could be used in optical-signal-processing applications.

\section{ACKNOWLEDGMENT}

This work was supported by the U.S. Department of Energy Office of Inertial Confinement Fusion under agreement No. DE-FC03-85DP402(V) and hy the Laser Fusion Feasibility Project at the Laboratory for Laser Energetic:s, which is sponsored by the New York State Energy Research and Development Authority and the Uriversity of Rochester. 


\section{REFERENCES}

1. A. M. Weiner, J. P. Heritage, and R. N. Thurston, Opt. Lett. 11, 153 (1986).

2. M. Haner. F. Spano, and W. S. Warren, in Ultrafast Phenomena V. edited by G. Fleming and A. Siegman (Springer-Verlag, New York, 1984), p. 514.

3. J. Nuckolls et al, Nature 239. 139 (1972).

4. S. C. Burkhart and R. B. Wilcox, IEEE Trans. Microwave Theory Tech. 28 $1514(199(3)$.

5. M. Haner and W. S. Warren, Appl. Phy's. Lett. 52. 1458 (1988).

6. A. M. Weiner, J. P. Heritage, and E. M. Kirschner, J. Opt. Soc. Am. B 5, 1563 (1988) and references therein.

7. A. A. Mak et al.. IAEA Technical Committee Meeting on Drivers for Inertial Confinement Fusion (Osaka, Japan, 1991).

8. S. W. Thomas, in High Speed Photography (SPIE, Bellingham. WA. 1976), Vol. 97.p. 73.

9. R. S. Marjoribanks, P. A, Jaanimagi, and M. C. Richardson, in High Speed Photography, Videography, and Photonics $V$ (SPIE. Bellingham, WA. 1986), Vol. 693, p. 134.

10. S. Skupsky, R. W. Short, T. Kessler, R. S. Craxton, S. Letzring, and J. M. Soures, J. Appl. Phys. 66, 3456 (1989).

11. A. M. Weiner et al., Opt. Letl. 15, 326 (1990).

12. E. B. Treacy, IEEE J. Quantum Electron. 5, 454 (1969).

13. M. Pessot, P. Maine, and G. Mourou, Opt Commun. 62,419(1987),

14. J. Desbois, F. Gires, and P. Tournois, IEEE J. Quantum Electron, 9, 21,3 (197.3).

15. J. Agostinelli et al., Appl. Opt. 18. 2500 (1979).

16. Y.-H. Chuang. Z.W. L. Li, D. D. Meyerhofer, and A. Schmid, Opt. Lett. 16, $7(1991)$.

\section{C Measuring Laser-Plasma X-Ray Emission Using Photodiode Arralys}

Traditionally, in laser-fusion and laser-plasma research, film has been used in $x$ ray diagnostics to record the integrated $x$-ray flux. The advantages of film are its low cost and relative simplicity of use. Photodiode arrays (PDA 's), although more complex, offer the advantages of rearly instantaneous tesults, high sensitivity, stability of response, and in principle are easy to calibrate. A PDA, manufactured by Hughes Aircraft in Carlsbad. CA, incorporated into a pinhole camera, has been used to image laser-plasma $x$ rays. The readout electronics were developed at the Space Science Laboratory and experiments were performed on the University of Roshester's OMEGA target chamber. 
$X$-ray imaging and $x$-ray spectroscopy are used in laser-fusion research to diagnose conditions in the targets. Examples of imaging diagnostics are pinhole cameras, Kirkpatrick-Baez microscopes, and Wolter microscopes. X-ray spectra can be obtained with various crystal or grating-dispersed spectrometers. The $x$-ray flux from these diagnostics can be recorded directly with film or by a solidstate imaging array such as a PDA, or a charged-coupled device (CCD). PDA's are more able to withstand the high instantaneous flux levels present in laserfusion experiments and are therefore more appropriate for use in direct $x$-ray detection. $X$ rayscan also be recorded indirectly. Indirect detection is accomplished by allowing the $x$ rays to impinge on a phosphor screen or a photocathode. If a phosphor alone is used, then visible-light photons are generated and subsequently detected. If a photocathode is used, then photoelectrons are generated. Photocathodes allow image-intensification techniques to be used. CCD's are often more appropriate for use in indirect imaging diagnostics since they have higher ultimate sensitivity because of their lower inherent noise.

If high spatial resolution is required, then direct detection of $x$ rays is often necessary. This is a result of the limited resolution obtainable with phosphors or photocathodes ( $>100 \mu \mathrm{m}$ is typical). In such a case, film or a solid-state array can spatially resolve the $x$-ray flux. The solid-state array, although considerably more complex and more expensive than film, offers the advantages of nearly instantaneous results, high sensitivity, and stability of response. Both PDA's and CCD's can be obtained with pixel sizes of $\sim 20 \mu \mathrm{m}$ and, therefore, comparable spatial resolution. If higher spatial resolution is needed, then film is still the only medium available since resolutions of belter than $5 \mu \mathrm{m}$ can be easily obtained with the finer-grained $x$-ray film.

A demonstration of the direct detection of $x$ rays has been performed with a hybrid Hughes PDA-readout chip combination. The applicability of the photodiode arrays manufactured by EG\&G Reticon is also examined.

\section{Detector Sensitivity}

The photodiode array has a dynamic range limited at the lower end by the background noise and at the higher end by the well depth of the diode. The sources of noise in PDA's are thermal, readout, and pattern noise. 'Both the thermal electron noise and the readout noise are functions of temperalure and so can be minimized by cooling the array. Sirice they have been extensively characterized, the Reticon S series are used as a point of comparison. Allinson et al.' have shown that for the Reticon RI.1024SF PDA, a practical lower limit to the total noise is $400 \mathrm{e}$. The well depth of the RL $1024 \mathrm{SF}$ is $9 \times 10^{7} \mathrm{e}$ yielding a theoretical dynamic range of $2 \times 10^{5}$ or $107 \mathrm{~dB}$. As configured by EG\&G Princeton Applied Research in the $x$-ray OMA 111 system, each photosite has a clear aperture of $2.5 \mu \mathrm{m} \times 2.0 \mathrm{~mm}$ and is covered with a $127-\mu \mathrm{m}$-thick. Be light shield. The useful range of detectable $x$-ray fiux levels can best be described by expressing the flux in units of energy-per-unit area. Since the energy per eleriron-hole pair in silicon is $3.7 \mathrm{eV}$, the well depth yields a maximum storable energy per readout of $3.3 \times 10^{8} \mathrm{eV}$ or $5.3 \times 10^{-4} \mathrm{ergs}$. Taken over the full aperture of the PDA this would yield a maximum detectable flux level of $1.1 \times 10^{-1} \mathrm{ergs} /$ $\mathrm{cm}^{2}$. However, a narrower aperture could be used in front of the array to increase 
Fin. 50.20)

Schematic of the $x$-ray pinhole camera system an deployed on the OMECBA target chamber. the maximum detectable flux level while the actual stored charge remained the same. As an example, a $25-\mu \mathrm{m}$ aperture would allow detection of flux levels up $108.5 \times 10^{1} \mathrm{ergs} / \mathrm{cm}^{2}$. The minimum detectable flux level in the context of laser fusion (where all photons are counted simultancously) is restricted to be approximately greater than 1 photon per pixel. At $8 \mathrm{keV}$ and using the full aperture of the PDA, this corresponds to a flux of $2.6 \times 10^{-5} \mathrm{ergs} / \mathrm{cm}^{2}$. So, by choosing the aperture appropriately, flux levels from $2.6 \times 10^{-5}$ to $8.5 \times 10^{1} \mathrm{ergs} /$ $\mathrm{cm}^{2}$ could be measured.

Two films commonly used for detecting laser-plasma x rays are Kodak 2495 and Kodak DEF. These have been characterized for their response to $x$ rays by Henke, 2,3 DEF is the more sensitive over the range from 1 to $10 \mathrm{keV}$. Goodman ef $a l .^{4}$ have compared the sensitivity of the OMA III PDA system to DEF. They find that the PDA has a useful dynamic range seven times greater than DEF film and has greatly superior low-flux performance.

\section{Experiments}

Experiments were carried out with a pinhole camera attached to the OMEGA laser target chamber. The detector was a Si photodiode array of $256 \times 256$ pixels, 30 $\mu \mathrm{m}$ on a side, with a $300-\mu \mathrm{m}$-thick depletion layer. The diode was indiumbump bonded to a Hughes readout device ${ }^{5}$ and the signal measured with custom, double-correlated, sample-and-hold electronics. ${ }^{6}$ Figure 50.20 shows a schematic of the arrangement used for these experiments. The detector was in its own vacuum housing, separated from the OMEGA chamber by a thin Be window (100) $\mu \mathrm{m}$ thick). A gate valve was placed between the detector housing and the OMEGA chamber so that the detector could be installed and removed while the OMEGA target chamber was under vacuum. A $1(0-\mu$ m-diam pinhole laser drilled into a $25-\mu \mathrm{m}$-thick tantalum foil wats used to image the $x$-ray emission. The pinhole alraty was placed at a distance of $19.0 \mathrm{~cm}$ from the target while the detector was placed at a distance of $95.0 \mathrm{~cm}$ from the pinhole array yielding a magnification of 5.0.

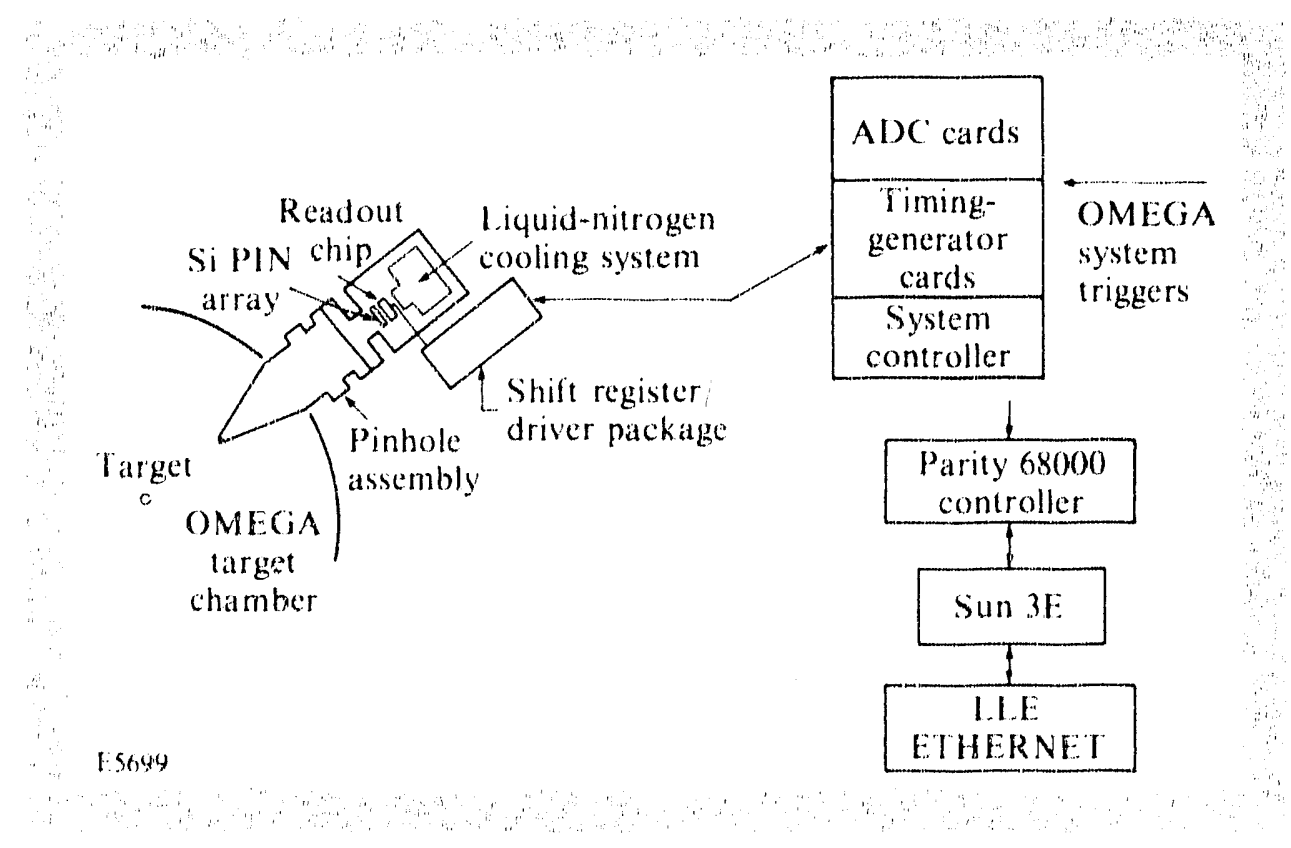


Timing of the detector readout was accomplished by sending a trigger signal to the readout electronics $45 \mathrm{~ms}$ before the laser shot. The readout cycle consisted of four full-frame readouts. The laser shot occurred during the second frame readout. Since the duration of the subsequent $x$-ray emission was short $(\sim 1 \mathrm{~ns})$, the frame was effectively recorded during the time of one pixel readout. Consequently, the image was recorded in the last part of frame 2 and the first part of frame 3. Frames 1 and 4 sampled the before and after shot background. The final background-corrected image was produced by adding frames 2 and 3 and subtracting frames 1 and 4 . The before and after background frames were examined for differences caused by incomplete recovery by the detector. No significant differences were seen.

Figure 50.21(a) shows an image obtained on a target shot where the beams were all surface focused. The target was an 80()$-\mu$ m-diam polystyrene sphere with a $1-\mu \mathrm{rn}$-gold overcoat. [The purpose of this target shot was to verify OMEGA beam targeting before beginning uniform or overlapping beam illumination of smaller $\left(\sim 250-\mu \mathrm{m}\right.$-diam) targets. $\left.{ }^{7}\right]$ Figure 50.21 (b) shows an $x$ ray image of the target taken by the nearest film-recording pinhole camera. This camera was located directly below the PDA detector on the target-chamber sphere. The $x$-ray emission regions produced by the focused OMEGA beams (beam spots) appear rotated downward on the PDA image relative to their locations on the film-recorded image. Note also that during the time of these experiments one beam was transported to the target through an auxiliary focus lens. The $x$-ray spot from this beam appears along the equator of the filmrecorded image at the right side [Fig. 50.21(b)]. This same spot appears $\sim 45^{\circ}$ clockwise from the apparent equator and near the limb of the target image |Fig. $50.21(a) 1$. The beam spot at the top of the PDA image shows an apparent break in the emission. This is a result of the emission being partially obscured by the stalk. This is nol seen on the film-recorded image since no beam spots seen from that view are obscured by the stalk. The relative size of the beam spots appears larger than the size of those seen with the filn because of the much higher sensitivity of the PDA, enabling the weaker emission from the edges of the beam spots to be detected by the PDA but not by the film. The centers of the PDA image appear uniform because the $x$-ray emission creates a saturated signal. [The Hughes array has a much lower well depth $\left(10^{6} \mathrm{e}^{--}\right)$than that of the Reticon PDA previously discussed. I

(a)

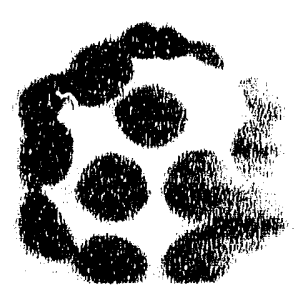

55712 (b)

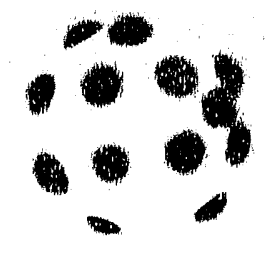

$X$ raly images of an OMEGA pointing shot: (a) from the PDA-recording pinhole camera; (b) from a film-recording pinhole camera. 


\section{Conclusions}

Photodiode arrays provide the experimentalist with an $x$-ray detection medium that is more sensitive than film and has a larger dynamic range than film. They are appropriate for use in imaging or spectroscopic diagnostics of laser-plasma $x$ rays. This has been demonstrated with the Hughes PDA attached to an $x$-ray pinhole camera deployed on the University of Rochester's OMEGA laser larget chamber.

\section{ACKNOWLEDGMENT}

This work wits supported by the U.S. Deparment of Energy Office of Inertial Confinement Fusion under agrement No. DE-FC(0.7-8.5DP4()20) and DE-AS(38-87DPIO685 and hy the Laser Fusion Feasibility Project at the Laboratory for Latser Energetics, which is sponsored by the New rork State Energy Research and Development Authority and the University of Rochester.

\section{REFERENCES}

1. N. M. Allinson et al., Nucl. Instrum. \& Methods Phys. Res. Sect. A 266. $592(1988)$

2. B. L. Henke et al., J. Opt. Soc. Am. B 1, 828 (1984).

3. B. L. Henke at al., J. Opt. Soc. Am. B 3, 1540 (1986).

4. D. A. Goodman er al., J. X-Ray Technol. 1, 162 (1989).

5. S. Gaalema, IEEE Trans. Nucl. Sci. 32, 417 (1985).

6. M. Peck er al., IEEE Trans. Nucl. Sci. 36, 950) (1989).

7. LLE Review 43, 142 (1990).

\section{D Ionization of Atoms with Intense, Linearly and Circularly Polarized, Picosecond Laser Pulses}

Current high-power lasers can produce focused intensities in which the electric lield is comparable to that which birds an atomic electron $\left(-10^{9} \mathrm{~V} / \mathrm{cm}\right)$. Under these conditions, the externally applied field cannot be treated as a small perturbation of the atomic system. The atomic-and laser-field systems must be considered together, opening an interesting new regime of atomic-physics research. An atom exposed to these strong fields becomes severely distorted and can undergo rapid ionization. The goal of this research is to study the response of atoms and ions to these extreme conditions.

A difficulty with experiments measuring the ionization of atoms and ions with high-intensity lasers has been the uncertainty in the absolute intensity, which is often a factor of 2 . This makes a comparison between theoretical predictions and experimental results some what imprecise and can make it difficult to distinguish different ionization mechanisms. On the other hand, the relative (shot-to-shot) laser intensity can be measured with significantly higher precision, perhaps $\pm 20 \%$ or better. Thus, comparisons between experimental results and theoretical predictions are facilitated by dependence upon the relative rather than absolute measurements of the iaser intensity. 
This article presents results of experiments comparing the intense field ionization of Xe and Ne aroms with linearly and circularly polarized, picosecond laser pulses. In a previously reported work, the ionization of noble gases was measured using a $1-\mathrm{ps}, 1-\mu \mathrm{m}$ wavelength laser with intensities up to mid$10^{16} \mathrm{~W} / \mathrm{cm}^{2}$ with linear polarization. ${ }^{1,2}$ It was found that the ionization threshold intensity of different charge states scaled as $E_{i o n}{ }^{4} / Z^{2}$ and that classical, fieldinduced ionization [barrier suppression ionization (BSI)] could best explain the experimental results over the large range of ion species measured. It was also found that a complex-atom, tunneling-ionization model of Ammosov, Delone, and Krainov (ADK $)^{3}$ could also explain the ionization of $\mathrm{Xe}$ and $\mathrm{Kr}$, though it did not work quite as well for the lighter noble gases. By comparing the ionization with linearly and circuarly polarized laser light, we are able to distinguish between the two theorie: and find that the tunneling theory (ADK) provides a more accurate descriptio' of the relative ion production for the two polarizations.

\section{Experimental Observations}

The laser used for these experiments is based on the chirped-pulse-amplification technique and has been described in detail elsewhere. ${ }^{4.5} \mathrm{CPA}$ laser systems allow the production of high-power, ultrashort pulses in solid-state lasing media. ${ }^{6}$ A laser pulse from a mode-locked oscillator is frequency chirped, temporally expanded in a fiber, and further stretched in time by an expansion grating pair. The longer pulse allows more energy to be extracted from the subsequent amplifier system than would be by a short pulse. After amplification, the pulse is compressed by a grating pair to picosecond or subpicosecond duration. There is a resulting increase in power by a factor equal to the chirp ratio (i.e., the stretched-pulse duration divided by the compressed-pulse duration). The chirping in the fiber, the compression and expansion grating pairs, and the chirp ratio are well described in Refs. 7--10.

For these experiments the compressed pulse was focused to a maximum intensity of approximately $1 \times 10^{15} \mathrm{~W} / \mathrm{cm}^{2}$ by a 20 - $\mathrm{cm}$ focal-length lens located inside a vacuum chamber. The chamber was backfilled with either xenon or neon 10 a known pressure between $5 \times 10^{-8}$ and $5 \times 10^{-5}$ Torr. A time-of-flight ion spectrometer was used to separate the ion species. The spectrometer has an extraction voltage of $800 \mathrm{~V} / \mathrm{cm}$ and a drift tube length of $60 \mathrm{~cm}$. The ion detector is a dual microchannel plate typically operated at $2 \mathrm{keV}$ for maximum gain. The ion signal is digitized with a 100-MHz transient digitizer (Kinetic Systems 4030) and the signals are stored digitally. Analysis of the spectra consists of integrating the various ion peaks to find the number of ions in each peak. The ion number for each laser shot is then plotled versus the incident laser intensity. A data run consists of taking approximately 150 laser shots at each of three different back fill pressures. The data taken at the highest intensities for linear polarization were laken with extra wire grids placed in the spectrometer to reduce the transmission efficiency. The use of different backfill pressures and the addition of the wire grids are necessary to avoid detector saturation for large ion number. Since the spectrometer efficiency is known, the data can be sealed to account for these changes. 
Fig. 50.22

Comparison of the production of $\mathrm{Xe}^{+}$and $\mathrm{Ne}+$ ions for linearly and circularly polarized light. The laser pulse length is $1.9 \mathrm{ps}$.
Data were taken using both linearly and circularly polarized light. The experiments with linear polarization were performed with a quarter-wave plate in the beam that was sef for zero retardation. When circular polarization was required, the wave plate was rotated by $45^{\circ}$. This ensures that any distortion in the wavefront or energy attenuation that might be introduced by the wave plate is present for both polarizations.

A comparison of the production of singly ionized xenon and neon for linearly and circularly polarized light is shown in Fig. 50.22. In the case of circular polarization an intensity increase of about $1.7 \pm 0.1$ is required to produce the same number of xenon ions as in the linear polarization case, and a factor of $1.5 \pm 0.1$ is required for neon.

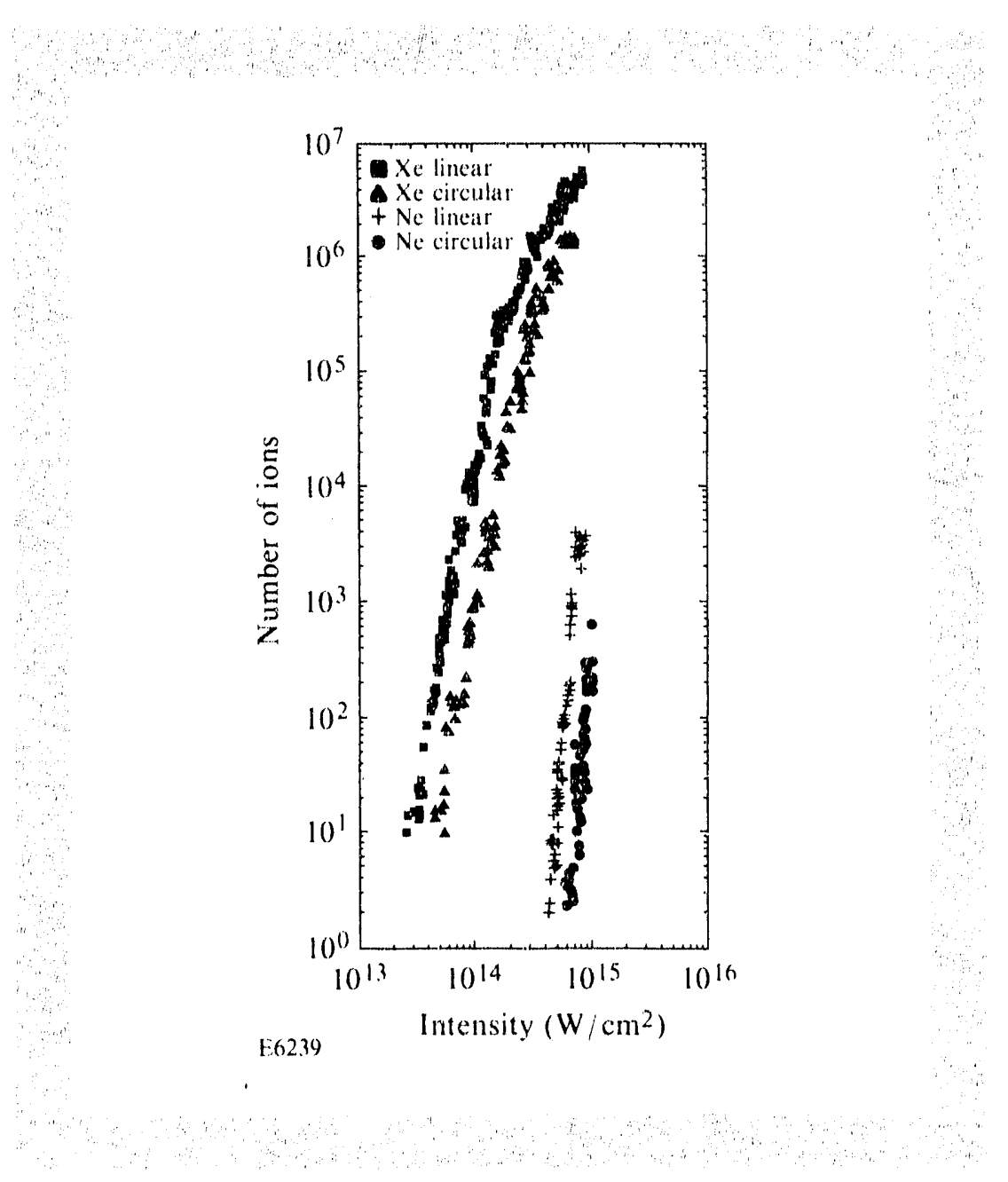

Theorefical Predictions
Experiments involving
traditionally been divided
former, laser ionizarion
latter, ionization can be de
be quantitatively differen

Experiments involving the interaction of atoms with intense laser fields have raditionally been divided into two regimes: multiphoton and tunneling. In the former, laser ionization can be described by perturbation theory, while in the be quantitatively differentiated by introducing the Keldysh $\gamma$ parameter ${ }^{11}$ 


$$
\gamma=\sqrt{\frac{E}{2 \Phi}},
$$

where $E$ is the ionization potential of the atom or ion and $\Phi$ is the ponderomotive potential of the laser. The ponderomotive potential is equal to the average kinetic energy of an electron oscillating in a laser field. The multiphoton regime has traditionally corresponded to $\gamma$ " with $\gamma$ \& 1 corresponding to the lunneling regime, in the tunneling regime, the electric field can be treated with a quasistatic approximation.

We have recently reported studies of ionization in the funneling regime of five noble gases using the $\mathrm{T}^{3}$ laser, ${ }^{1.2}$ where a comparison between our data and various theoretical models for photoionization was made. Two theories agreed well with the experimental data. Both of these are explicitly based on a quasistatic model of the laser field.

BSI is a simple, one-dimensional model of ionization ${ }^{1,12}$ based on the superposition of the Coulomb potential and a quasi-static laser field. The threshold intensity lor ionization $\left(l_{t h}\right)$ is defined as the intensity at which the peak clectric field allows the bound electron to escape without tunneling and is $I_{t h}=E^{4} /\left(16 Z^{2}\right)$ in atomic units, where $Z$ is the charge state of the atom or ion after jonization.

We have defined an experimental threshold intensity for ionization to be that intensity at which the ionization probability in the pulse is approximately $1 \%$. Figure 50.23 shows a comparison of the experimental and BSI ionization threshold intensities. As can be seen, there is good agreement between the model and the experiment over three orders of magnitude in laser intensity.

Fig. 5() .23

Comparison of the measured threshold intensity for ionization of various charge states of a noble gas with the BSI predictions (from Ref. 1).

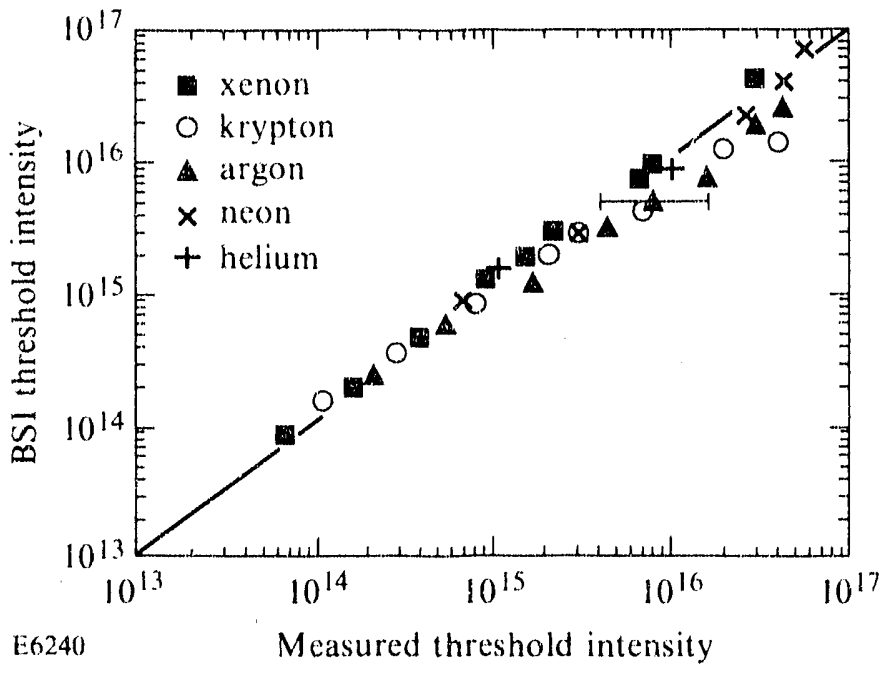


The ADK model. ${ }^{3}$ is an extension of the 3-dimensional (3-D) de-tunneling model. The 3-1), de-lunneling model supplies the tunneling ionization rate for a hydrogen atom in a static electric field and is given by 13,14

$$
W_{i}=4 \omega_{A} \frac{\left(2 E_{i}\right)^{5 / 2}}{\varepsilon} \exp \left[-\frac{2\left(2 E_{i}\right)^{3 / 2}}{3 \varepsilon}\right] .
$$

Perelonov et al. ${ }^{15}$ introduced an atomic shell dependence and an oscillatory electric field to this model, resulting in an ionization rate of

$$
\begin{aligned}
& W_{\text {lineir }}=\omega_{A} C_{n_{i}{ }^{*},} f_{i}(l, m) E_{i}\left[\frac{3 \varepsilon}{\pi\left(2 E_{i}\right)^{3 / 2}}\right]^{1 / 2} \\
& {\left[\frac{2}{\varepsilon}\left(2 E_{i}\right)^{3 / 2}\right]^{2 n n_{i}^{*}-|m|-1} \times \exp \left[-\frac{2}{3 \varepsilon}\left(2 E_{i}\right)^{3 / 2}\right] .}
\end{aligned}
$$

where the factor $f$ is given by

$$
f(1, m)=\frac{(2 l+1)(1+|m|) !}{2^{|m|}(|m|) !(1-|m|) !} .
$$

The term $\left[3 \varepsilon / \pi(2 E)^{3 / 2}\right]^{1 / 2}$ in Eq. (3) results from averaging over one period of the laser, and for the lasser parameters discussed here, it is typically of order 10 ' . Atomic structure is introduced through the factors $f(l, m)$ and $C_{n^{*}}$, but Perelomov et a $1 .{ }^{15}$ did not derive an expression for the $C_{n n^{*}}$ 's. Ammosov et al. ${ }^{3}$ extended the theory by deriving an approximate expression for the $C_{n} * 1$ s. This wats accomplished by joining the asymptotic wave function of the free electron with the quasiclassical radial wave function of a bound electron. ${ }^{13}$ The initial atomic or ionic state is described by the effective principal quantum number $n^{*}$, the orbilal angular momentum, and the magnetic quantum numbers $/$ and $m$. A species-dependent jonization rate results from this inclusion of $n^{*}$ in the theory. The expression for $C_{n n^{*} /}$ is given by Ammosov as ${ }^{3}$

$$
C_{n * l}=\left(\frac{2 e}{n *}\right)^{n *} \frac{1}{(2 \pi n *)^{1 / 2}}
$$

where ${ }^{\prime}=2.71828 \ldots$ For ionization rate calculations, we have used the ground state values ${ }^{16}$ for $n^{*}$ and $l$ and a summation over the degenerate $m$ states.

Equation (3) is an approximation valid in the limits $n^{*}$ " $1, \varepsilon_{\text {laser }}$ " $\varepsilon_{\text {binding, }}$ and $\omega_{t}$ " $E$. The validity of the ADK is expected to improve as $n^{*}$ increases, and 
larger $n^{*}$ occurs for heavier atoms and higher charge states. Thus, the agreement is expected to be best for xenon and worst for helium.

It was found that ADK and BSI theories both predict the correct spacing for all charge states observed ( $E^{4} / Z^{2}$ dependence), but the fit between the ADK theory and the data from the lighter noble gases (hclium, neon, and argon) had an absolute error in the threshold intensities of 2 or less. $^{2}$ The agreement with ADK improved for increasing atomic number since $n^{*}$ is also increasing, and the slope of the ion curve for low ion number agrees with the experiment better than the BSI theory. It should be noted that the absolute error in threshold intensities that ADK predicts for the lighter noble gases is never larger than the experimental uncertainty present in our absolute intensity measurements (a factor of 2), but it is larger than the relative uncertainty.

\section{Circular versus Linear Polarization}

Both BSI and ADK models of ionization depend on the strength of the electric field. For linearly polarized light, the electric field oscillates in time, $\varepsilon(t)=$ $e_{1} \cos \omega t$, and is related to the intensity as $l_{1}=\varepsilon_{1}{ }^{2}$, whereas for circularty polarized light, the electric field has a constant amplitude and is related to the intensity as $I_{c}=2 \varepsilon_{c^{\prime}}{ }^{2}$. In this section, the intensity dependence of the two ionization models is considered by comparing the relative field strengths at which linear and circular polarization give the same ionization rates. At the same ionization rate linear and circular polarization will yield the same number of ions.

Classical field ionization (BSI) depends only on the strength of the electric field and is independent of the oscillatory nature of the field as long as the oscillation frequency is much less than the atomic orbital frequency. Thus for BSI, one expects a factor-of- 2 increase in intensity for ionization by circular polarization compared to linear, to produce the same number of ions.

For tunneling ionization, the picture is a little more complicated. At the same intensity, the field strength for circular polarization is $\sqrt{2}$ smaller than that for linear; this reduces the tunneling rate. On the other hand, the circularly polarized field is on continuously; thus, the static field rate

$$
\begin{gathered}
W_{\text {circular }}=\omega_{A} C_{n_{i}^{*} l}^{2} f_{i}(l, m) E_{i} \\
{\left[\frac{2}{\varepsilon}\left(2 E_{i}\right)^{3 / 2}\right]^{2 n_{i}^{*}-\{m i-1} \times \exp \left[-\frac{2}{3 \varepsilon}\left(2 E_{i}\right)^{3 / 2}\right],}
\end{gathered}
$$

rather than the time averaged rate (Eq. 3), is applicable. The omission of the averaging term $\left[3 \varepsilon / \pi(2 E)^{3 / 2}\right]^{1 / 2}$ decreases the intensity difference to less than a factor of 2. By comparing the linear and circular ionization rates in the intensity ranges of the experiments, we find that the intensity required to ionize $X e$ atoms should be a factor of $1.6 \pm 0.1$ times higher for circular polarization than for linear and a factor of $1.5 \pm 0.1$ for Ne atoms, which is consistent with the experimental results. 


\section{Conclusions}

In a previous experiment, it was found that both BSI and ADK could be used to explain the high-field ionization of noble gases. ${ }^{2}$ Both theories show a $E_{\mathrm{i} o n}{ }^{4} / Z^{2}$ dependence in the ionization thresholds of the charge states within the absolute uncertainty in the laser intensity, although ADK systematically uncerestimated the ionization rate for the lighter noble gases. By comparing ionization with circular and linear polarization, it is found that the ADK tunneling model produces good agreement with the experimental results, while BSI overestimates the required circularly polarized intensity.

\section{ACKNOWLEDGMENT}

This work is supported by the National Science Foundation under contract No, PHY 8822730 Idditional support is provided by the U.S. Departi, ant of Energy Office of Inertial Confinement litusion under agreement No. DE-FC(3-8.5DP4(20) and by the Laser Fusion Feasibility Project at the Laboratory for Latser Energeties, which is sponsored by the New York State Energy Research and Development Authority and the University of Rochester.

\section{REFERENCES}

1. S. Augst, D. Strickland, D. D. Meyerhofer, S. L. Chin, and J. H. Eberly, Phys. Rev. Lett. 63, 22.12 (1989).

2. S. Augst, D. D. Meyerhofer, D. Strickland, and S. L. Chin, J. Opt. Soc. Am. B 8, 858 (1991).

3. M. V. Ammosov, N. B. Delone, and V. P. Krainov, Sov. Phys. JETP 64, 1191 (1986).

4. P. Maine, D. Strickland, P. Bado, M. Pessot, and G. Mourou, IEEE J. Quantum Electron. 24, 398 (1988).

5. Y.-H. Chuang, D. D. Meyerhofer, S. Augst, H. Chen, J. Peatross, and S. Uchida, J. Opt. Soc. Am. B 8, 12.26-1235 (1991).

6. D. Strickland and G. Mourou, Opt. Commun. 56, 219 (1985).

7. A. E. Siegman, Lasers (University Science Books, Mill Valley, CA, 1986), pp. 331-361; ibid. pp. 1171-1212.

8. E. B. Treacy, IEEE J. Quantum Electron. 5, 454 (1969).

9. M. Pessot, P. Maine, and G. Mourou, Opt. Commun. 62, 419 (1987).

10. G. P. Agrawal, Nonlinear Fiber Optics (Academic Press, Boston, 1989), pp. $1-342$.

11. L.V. Keldysh, Sov. Phys. JETP 20, 1307 (1965).

12. H. A. Bethe and E. E. Salpeter, Quantum Mechanics of One- and Two-Electron Atoms (Plenum Press, New York, 1977).

13. L. D. Landau and E. M. Lifshitz, Quantum Mechanics (Pergamon, New York, 1965).

14. P. B. Corkum, N. H. Burnett, and F. Brunel, Phys. Rev. Lett. 62, 1259 (1989). 
15. A. M. Perelomov, V. S. Popov, und M. V. Terent'ev, Sov. Phys. JETP 23, 924 (1966).

16. R. L. Kelly and L. J. Palumbo, Atomic and Ionic Emission Lines Below'2000 Angstroms: Hydrogen Through Krypton (U.S. Govt. Print. Off., Naval Research Laboratory, Washington, DC, 1973).

\section{E Femtosecond Nonlinearities and Hot-Carrier Dynamics in GaAs}

The properties of hot carriers are determined primarily by the electronic band structure of the host material and carrier-lattice and carrier-carrier interactionstopics that have historically been of sustained interest in solid-state physics. Because of the continuing size reduction of conventional semiconductor devices and the emergence of new devices based upon advanced growth techniques, the study of hot-carrier dynamics remains of importance. Current research on hotcarrier dynamics relies on optical techniques, often using light both as an injector of relatively mono-energetic electrons and as a probe of the carrier distribution. The advent of ultrashort laser pulses provides a very direct means of investigating hot-carrier relaxation and cooling processes with excellent temporal resolution.

Extensive investigations of hot-carrier dynamics in GaAs have been performed using ultrafast spectroscopy. ${ }^{1-14}$ In this article, we present a new series of studies of hot-carrier dynamics. The transient absorptive and refractive nonlinearities of GaAs have been measured with a temporal resolution of less than $100 \mathrm{fs}$. Various hot-carrier processes and their influences on the ultrafast optical response are discussed.

\section{Techniques}

We have used a copper-vapor, laser-amplified CPM laser to perform pumpprobe measurements on GaAs at room temperature. About 3\% of the 75- to 100 -fs pulses at $620 \mathrm{~nm}$ is used as the pump beam. The remainder is focused onto a jet of ethylene glycol, producing a white-light continuum, which is used as a probe pulse. The probe wavelength is selected with interference filters over a spectral region from $550 \mathrm{~nm}$ to $950 \mathrm{~nm}$. The polarization of the pump and probe pulses is orthogonal to reduce coherent artifacts. The intensity of the pump pulse can be varied over nearly two orders of magnitude.

The time-resolved transmission $T^{\prime}(t)$ and reflection $R(t)$ are measured simultaneously on a thin $(<0.3-\mu \mathrm{m})$ intrinsic GaAs film obtained using a lift-off technique ${ }^{15}$ and attached to a sapphire window. The absorption coefficient $\alpha(t)$ and refractive index $n(t)$ are then deduced from the measured $T$ and $R$ by inversion of the Fabry-Perot formulae. The changes of refractive index $\Delta n(t)$ are also obtained by measuring the time-resolved differential reflection $\Delta R / R$ on thick GaAs samples. ${ }^{13}$ 
Fig. 50.24

Measured $\Delta n$ spectrum for $N \sim 2.5 \pm 1.2 \times 10^{18}$ $\mathrm{cm}^{-3}$ at time delays: (a) $\mathrm{i} \sim()^{+}$and (b) $1 \sim$ 20()$^{+} \mathrm{fs}$. Squares are the measured average values for both a tilm and a bulk sample; circles are the measured average values for a thin film. The error bars are obtained from the standard deviation of the measured values and a conservative estimate of the uncertainty in carrier density. A clear spectral resonance around $620 \mathrm{~nm}$ appears in (a).

\section{Results}

1. Refractive-Index, Spectral-Hole Burning

For excitation of GaAs at $2 \mathrm{eV}(620 \mathrm{~nm})$, the electrons are initially injected into the $\Gamma$ valley at the three distinct excess energies of $0.50 \mathrm{eV}, 0.43 \mathrm{eV}$, and $0.15 \mathrm{eV}$, caused by transitions from the heavy-hole, light-hole, and split-off valence bands with a relative strength of $42 \%, 42 \%$, and $16 \%$, respectively. The transient decrease of absorption, caused by the nonthermal carrier distribution generated after femtosecond excitation, is characterized by an absorption spectral hole near $2 \mathrm{eV} .{ }^{6.16}$ According to the Kramers-Kronig relation, the existence of a spectral hole in the imaginary part of the refractive index (absorption) will cause a spectral resonance in the change of a real part of the refractive index. We have indeed observed this resonance around $2 \mathrm{eV}$, which we call refractiveindex, spectral-hole burning. ${ }^{13}$ Figure 50.24 shows ciur measurements of the spectral dependence of the refractive-index change foi $N \sim 2.5 \times 10^{18} \mathrm{~cm}^{-3}$. As shown in Fig. 50.24(b), the spectral hole disappears quickly because a large fraction of the electrons scatter to the $X$ and $L$ valleys and the rest thermalize within the $\Gamma$ valley on a sub-100-fs time scale.

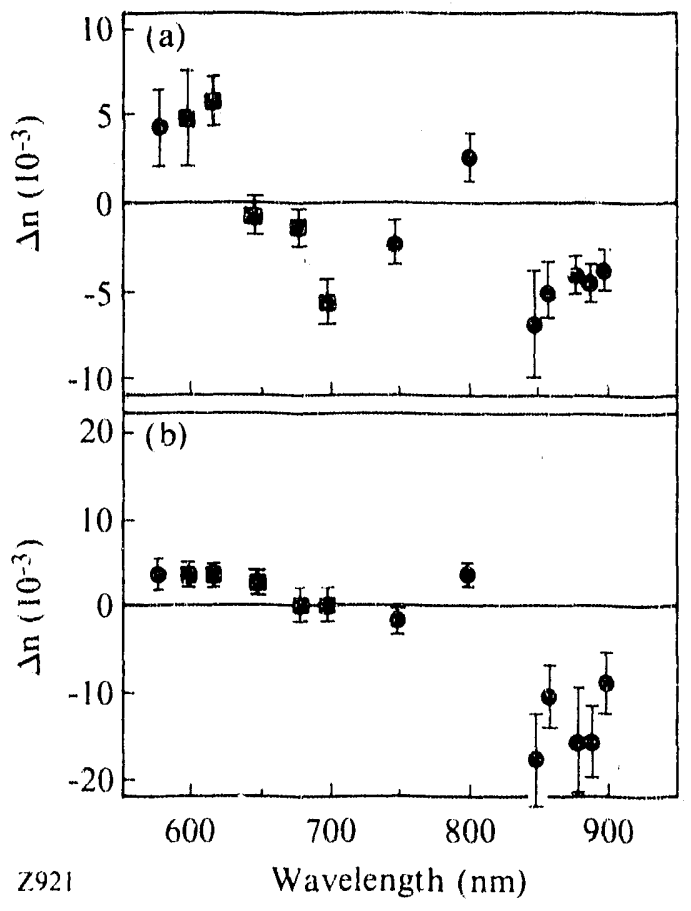

If the absorption spectral hole burned at $t \sim 0^{+}$followed the symmetric pump spectrum around $620 \mathrm{~nm}(2 \mathrm{eV})$, we expect that the change of the refractive index would be zero at $620 \mathrm{~nm}$. However, our data clearly show that the zero change of the refractive index is near $650 \mathrm{~nm}$, and not at $620 \mathrm{~nm}$. Furthermore, measurements on the absorption changes also confirm that at $1 \sim 0^{+},|\Delta \alpha|$ is larger near $650 \mathrm{~nm}$ than at $620 \mathrm{~nm}$, a result than can also be inferred from absorption 
spectral-hole-burning measurements made around $620 \mathrm{~nm}$ by others. ${ }^{16}$ Therefore, on a time scale shorter than our temporal insolution, the initial excited carrier "distribution" is strongly deformed and becomes spectrally asymmetric, and the peak of the distribution appears to be red-shifted. The $\Gamma$-valley electrons excited from the heavy-hole valence band by $2-\mathrm{eV}$ photons can scatter to both $X$ and $L$ valleys; by contrast, those electrons excited from the light-hole valence band can only scatter to the $L$ valley. Therefore, the electrons excited from the heavyhole valence band are more likely to be transferred to the satellite valleys than those excited from the light-hole valence band. This "preferred" scattering produces a deformation of the excited.carrier distribution. This process also appears to be instantaneous within our temporal resolution because carriers excited by the earlier part of the pump pulse have already started undergoing this effect. Another effect, which was recently observed and termed "resonant intervalley scattering" by Bigot et al. ${ }^{17}$ can also contribute to the ultrafast deformation (shift) of the absorption spectral hole. Because of the vanishing density of $|X\rangle$ states at the $\Gamma-X$ transition point, the return of electrons from the bottom of the $X$ valley to the $\Gamma$ valley is more probable, thereby enhancing the concentration of electrons in the $\Gamma$ valley near the transition point. We note that the peak of the "trapped" electron distribution $(\sim 1.92 \mathrm{eV}$ or $646 \mathrm{~nm}, 30 \mathrm{meV}$ below the $X$-valley minimum) is very close to the zero change of the refractiveindex spectral resonance. It is still not clear why this resonant process takes place in $\sim 50 \mathrm{fs}^{17}$ because an electron has to emit or absorb a phonon twice in order to complete this process. Nevertheless, this explanation is supported by the following facts: the shift has little carrier-density dependence; it does not appear ${ }^{3}$ or is very smal! ${ }^{18}$ when the spectral hole is burned below the $L$-valley minimum; it does not appear in $\mathrm{InP},{ }^{19}$ a material in which there is no intervalley scattering with 620-nm excitation; and $\Delta n$ is nearly zero at the pump wavelength $(620 \mathrm{~nm})$ at $t \sim 0+$ for GaAs at low temperature $(2 \mathrm{~K})^{20}$ when the $\Gamma-X$ transition is suppressed.

2. Studies of the Initial Scattering Time

If pump-probe measurements are performed at the same wavelength, the recovery of the bleaching measures the scattering rate of carriers from the initially optically coupled states. Since $\Delta n(t)$ measures the spectrally integrated population change near the as-excited states, it is best to use $\Delta \alpha(t)$ to deduce the initial scattering time. Figure 50.25 shows $\Delta \alpha(t)$ at $620 \mathrm{~nm}$ for various injectedcarrier densities.

We have developed a simplified model in which the initial fast bleaching is modeled by two equations

$$
\begin{aligned}
& \frac{d N}{d t}=\frac{N_{\mathrm{o}} I_{\text {pump }}(t)}{\int_{-\infty}^{+\infty} I_{\text {pump }}(t) d t}-\frac{N}{\tau_{T}} \\
& S(t) \propto \int_{-\infty}^{+\infty} I_{\text {probe }}\left(t^{\prime}-t\right) N\left(t^{\prime}\right) d t,
\end{aligned}
$$


Fig. 50.25

'Time evolution of $\Delta O$ at $620 \mathrm{~nm}$ for different $N \cdots:$ (A) $3.3 \times 10^{18} \mathrm{~cm}^{-3}$, (B) $9.5 \times 100^{17} \mathrm{~cm}^{-3}$. and (C) $3 \times 10^{17} \mathrm{~cm}^{\cdots}$. where $N_{\mathrm{o}}$ is the total injected-carrier density, $N$ is the time-dependent c irier density in the excited states, $1 / \tau_{T}$ is an effective total scattering rate fro $: n$ the initial excited states, $I_{\text {purnp }}(t)=J_{\text {probe }}(t)=\operatorname{sech}^{2}\left(1.763 t / \tau_{p}\right)$, and $S(t)$ is the detected signal. If $\tau_{T} \gg \tau_{p}$, the decay part of $N(t)$ can be simply described by an exponential with time constant $\tau_{T}$. However, when $\tau_{T} \sim \tau_{p}$, as in our measurements, $N(t)$ does not have a simple form.

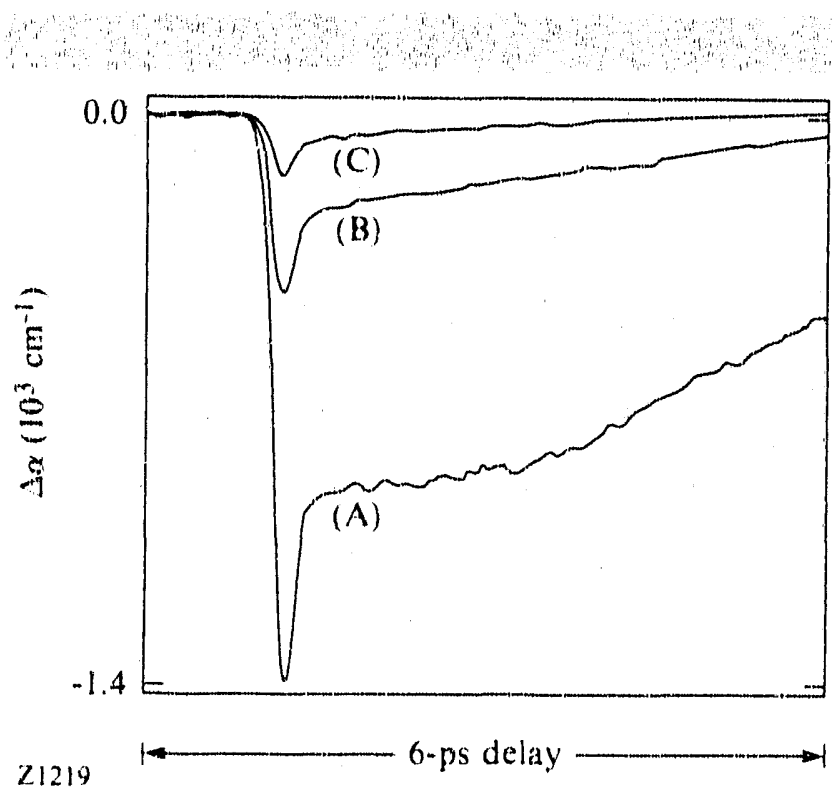

From Fig. 50.25, it is clear that the change of the signal is not governed by a single decaly process, but rather that a fast process is superimposed on a much slower variation. It is important to note that the slow variation is rather different at each carrier density. This result prevents the traces obtained for different carrier densities from being normalized by the amplitudes of their slow variations, a method used by others. ${ }^{6}$ However, at least for low carrier densities $\left(N<5 \times 10^{17}\right.$ $\left.\mathrm{cm}^{-3}\right)$. the slow variation of $\Delta \alpha(t)$ is well described by an exponential decay. The solid line shown in Fig $50.26(a)$ is a fit of the slow decay of $\Delta Q(f)$ for $N-3 \times 10^{17}$ $\mathrm{cm}^{-3}$ using Eys. (1) and (2) with $\tau_{p}=100 \mathrm{fs}$ and $\tau_{72}=2 \mathrm{ps}$. This slow component is subtratcted from the experimental trace to obtain the "effective" fast bleaching component. as shown by a dotted line in Fig. 50.26(b). We then use Eqs. (1) and (2) again to fil the fast bleaching. The solid line shown in Fig. $50.26(\mathrm{~b})$ is the best fir using $\tau_{p}=1(K)$ fs and $\tau_{71}=50$ ) $\mathrm{s}$.

It is worth mentioning that only less than $35 \%$ of the total excited electrons ever accumulate in the "as-excited" states; most of them have already scattered away within the pulse width since $\tau_{T 1} \sim 0.5 \tau_{p}$. After $\left.\sim 200\right)$ fs, the split-off probe makes a major contribution to $\Delta x$; it monitors the band filling and the cooling of the $\Gamma$-valley electron distribution $-1.50 \mathrm{meV}$ above the conduction-band edge. The fact that the split-off probe samples the Boltzmann "tail" for low carrier densities $\left(<5 \times 10^{17} \mathrm{~cm}^{-3}\right)$ is probably responsible for the exponential decay of the slow component. 
Fig. 50.26

(a) Measured time-resolved $\Delta \alpha$ at $620 \mathrm{~nm}$ for $N \sim 3 \times 10^{17} \mathrm{~cm}^{-3}$ (dotted line) and a fit (solid line) for a slow uecay with $\tau_{p}=100 \mathrm{fs}$ and $\tau_{T 2}=2 \mathrm{ps}$ : (b) The fast component (dotted line), obtained by subtracting a slow component / solid line in (a)] from the experimental curve [dotted line in (a)). is compared to different fits with $\tau_{p}=100 \mathrm{fs}$ and $\tau_{T 1}=50 \mathrm{fs}$ (solid line), $\tau_{T}=$ 70 fs (dashed line), and $t_{T}=30$ ) $\mathrm{s}$ (dashed-dotted line). The best fit is obtained using $\tau_{\eta}=50 \mathrm{fs}$.
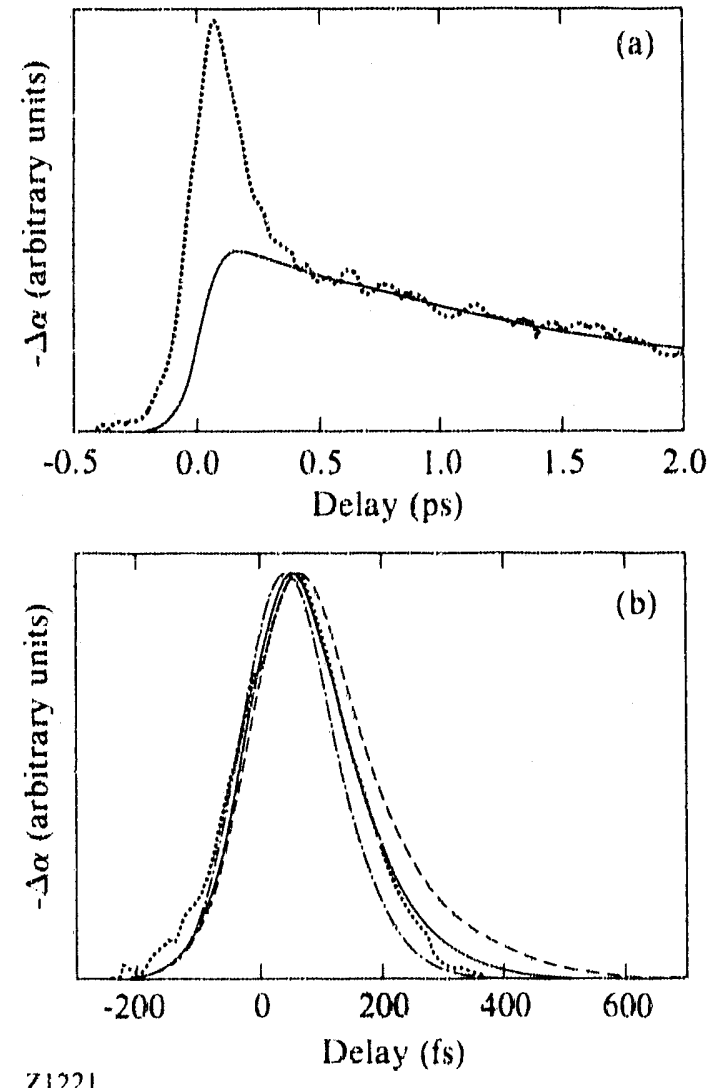

$2\{22\}$

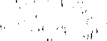

At higher carrier densities, the slow component is rather complicated and the simple fir described previously does not work well. However, information on the initial effective scattering rate can be obtained by monitoring the peak value of the transient spike of $\Delta \alpha(t)$ as a function of carrier density. Figure 50.27 plots $\left|\Delta \alpha_{\text {max }}\right|$ versus $N$. The weak sublinearity of the data indicates that $\tau_{T}$ decreases little at higher carrier densities: $\tau_{T}$ is reduced from $\sim .50$ fs $10 \sim 40$ fs when $N$ increases from $3 \times 10^{17} \mathrm{~cm}^{-3}$ to $7 \times 10^{18} \mathrm{~cm}^{-3}$. The value of $\tau_{r}$ at high $N(\cdots 40 \mathrm{fs})$ is very close to the values obtained by other groups with a three-timecomponent fit ${ }^{2,9}$ or a similar two-time component fit. ${ }^{21}$

We now consider the microscopic origin of the ultrafast initial scattering time. The contributions by electrons excited from the heavy-hole, light-hole, and splitoff valence bands all need to be considered. Intervalley scattering is a very important process, and its rate is currenly under investigation. ${ }^{22}$ Recently Zollner et al. ${ }^{23}$ introduced the concept of an effective intervalley deformation potential (IDP) in which the contribution of the TA phonons is included in a temperature-dependent IDP for LO-phonon scattering. If we use values of Zollner ef $a l .\left(D_{T L} \sim 5.8 \times 10^{8} \mathrm{eV} / \mathrm{cm}\right.$ and $D_{\Gamma X} \sim 9.4 \times 10^{8} \mathrm{eV} / \mathrm{cm}$ at $\left.T_{L}=300\right) \mathrm{K}$ ) to calculate the intervalley scattering times at different excited energies, we

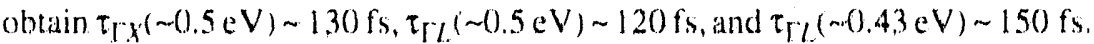


Fig. 50.27

Aamex $\mid\left(r \sim 0^{+}\right)$as a function of $N$ at $620 \mathrm{~nm}$. The straight line represents a linear relationship between $\left|A \alpha_{\max }\right|$ and $N$. $\tau_{\text {LOO }}$ (the unscreened LO-phonon emission time) is taken to be $\sim 180 \mathrm{fs} .^{4,24}$ The effective initial scattering lime is then given by

$$
\begin{aligned}
& \frac{1}{\tau_{T}}=0.42\left(\frac{1}{\tau_{\Gamma X}(0.5 \mathrm{eV})}+\frac{1}{\tau_{\Gamma L I}(0.5 \mathrm{eV})}+\frac{1}{\tau_{L . O}}\right) \\
& \quad+0.42\left(\frac{1}{\tau_{\Gamma L L}(0.43 \mathrm{eV})}+\frac{1}{\tau_{L O}}\right)+0.16\left(\frac{1}{\tau_{L O}}\right) .
\end{aligned}
$$

The calculated value of $65 \mathrm{fs}$ is close to the measured $\tau_{T} \sim 50 \mathrm{fs}$ at $N \sim 3 \times 10^{17}$ $\mathrm{cm}^{-3}$. The $30 \%$ difference can be attributed to carrier-carrier scattering. The fact that $\tau_{T}$ only decreases to 40 fs at high $N$ points out that carrier-carrier scattering does not strongly affect the initial scattering time, at conclusion also reached by others. ${ }^{20}$

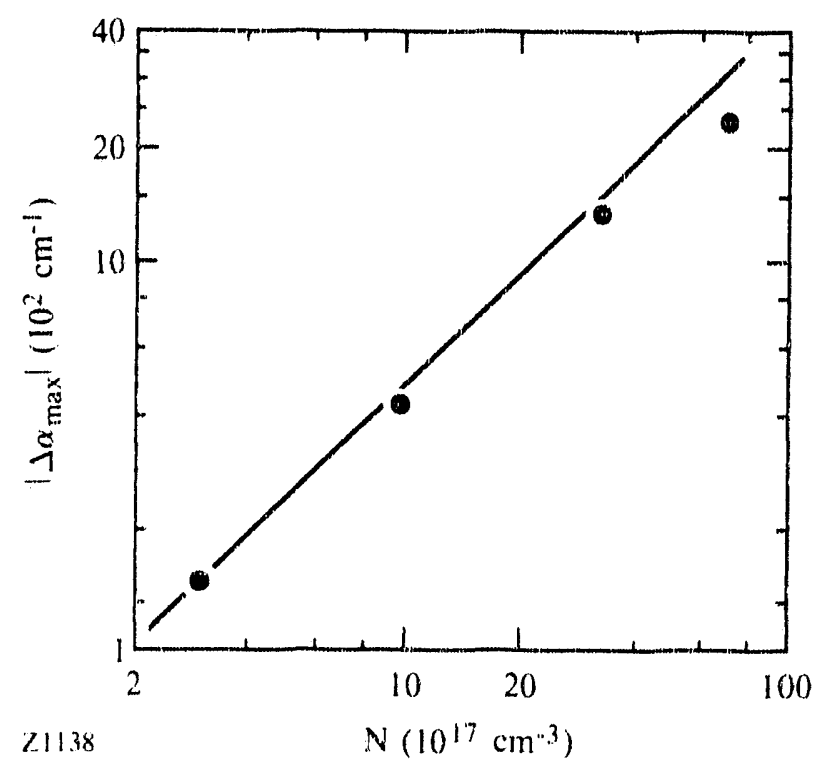

3. Band-Edge Nonlinearities

The interactions between hot carriers strongly affect absorptive and refractive nonlinearities around the band edge. Figare 50.28 shows the time-resolved changes of absorption for the same carrier density $\left(N \cdots 1.5 \pm 0.7 \times 10^{18} \mathrm{~cm}^{-3}\right)$ at probe wavelengths of $880,890,900$. and $920 \mathrm{~mm}$, which are below the band edge, and of $860 \mathrm{~nm}$, which is slightly above the band edge. The short- lived increase of absorption observed at 880,890 , and $9(00) \mathrm{nm}$ riglat after excitation is attributed 
Fig. 50.28

Measured, time-resolved $\Delta a$ for $N$. $1.5 \pm 0.7 \times 10^{18} \mathrm{~cm}^{\cdots} 3$. The probe wavelengths are: (A) $860 \mathrm{~nm}$, (B) $880 \mathrm{~mm}$, (C) $890 \mathrm{~nm}$, (D) $9(0) \mathrm{nm}$, and (E) $920 \mathrm{~nm}$. Curve (E) has been multiplied by 5 . to band-gap renomalization accompanied by plasma screening of Coulomb interactions. Coulomb screening is often neglected in the interpretation of many experiments. The decrease of absorption slightly above the band edge $(850$ and $860 \mathrm{~mm}$ ) immediately after excitation, which is caused by the reduction of Coulomb (Sommerfeld) enhancement factor, reveals the importance of this effect. Plasma screening of Coulornb interactions, along with band-gap renormalization, causes a clear spectral resonance in $\Delta \alpha$ around the band edge, which lakes place instantaneously when most carriers are still "hot". "The sur.sequent broadband decrease of absorption indicates that the states near the perturbed band edge become filled.

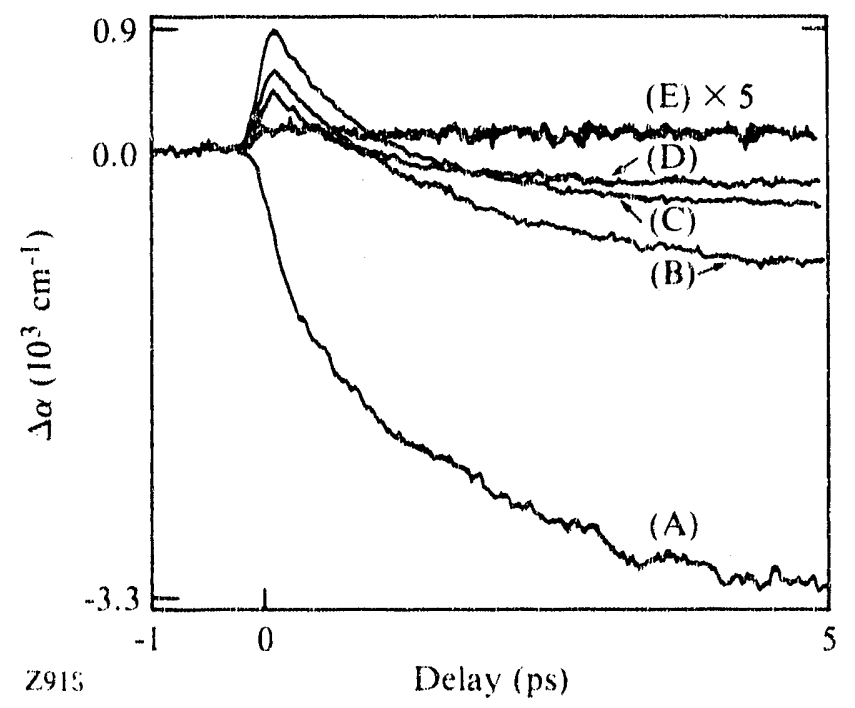

Al $920 \mathrm{~nm}$ ( $75 \mathrm{meV}$ below the original band edge), a small, long-lived induced absorption is observed. This affect is attributed to intra-band (free(arrier) absorption. We investigate it by probing at $950 \mathrm{~nm}$, which is $\sim 120 \mathrm{meV}$ below the original band edge. Figure $50.29(\mathrm{a})$ and $50.29(\mathrm{~b})$ show $\Delta \alpha(t)$ and $\Delta n(t)$ forvarious (high) carrier densities. Both $\left|\Delta \alpha_{\text {max }}\right|$ and $\left|\Delta n_{\text {max }}\right|$ scale approximately linearly with $N$, consistent with free-carrier absorption (FCA) being the main contribution to the nonlinearities at this wavelength. The cross section for FCA, defined as $\sigma_{c^{\prime} h}=\Delta \alpha / N_{+}$is $2.6 \pm 1.0 \times 10^{-17} \mathrm{~cm}^{2}$ at $950 \mathrm{~nm}$. Free-carrier absorption in 1 -type GaAs has been systematically studied earlier, ${ }^{25}$ and $\sigma_{\ell^{\prime}}$ (solely because of electrons) deduced from those data is $-8 \times 10^{-18} \mathrm{~cm}^{2}$. Other measurements 25,26 also show that $\sigma_{h}$ (solely caused by holes) is more than a factor of 2 larger than $\sigma_{e}$. Our estimated $\sigma_{e h}$ is close to $\sigma_{l}+\sigma_{h}$. It is interesting to note that $-5 \mathrm{ps}$ after excitation, when the carriers are cooled down, $\Delta \alpha$ is only $-20 \%$ smaller than after $<() .5 \mathrm{ps}$, when the carriers are still hof 27 and many of them reside in the satellite valleys. Also, $950 \mathrm{~nm}|\Delta n|$ is quite large because band filling causes a large negative $\Delta n$, which extends far below the band edge. ${ }^{28}$ Such large refractive nonlinearities should be useful for designing optoelectronic: devices such as phase modulators. 


\section{Fing. $5(1.29$}

Measured lime-resolved (a) $\Delta(x$ and (b) $\Delta / 1$ at 1) $50 \mathrm{~mm}$ for various $N \sim($ A $) 1.3 \times 1019$ $\mathrm{cm}^{-3},(B), 5 \times 10^{18} \mathrm{~cm}^{-3}$, (C) $2 \times 10^{18} \mathrm{~cm}^{-3}$. (a)

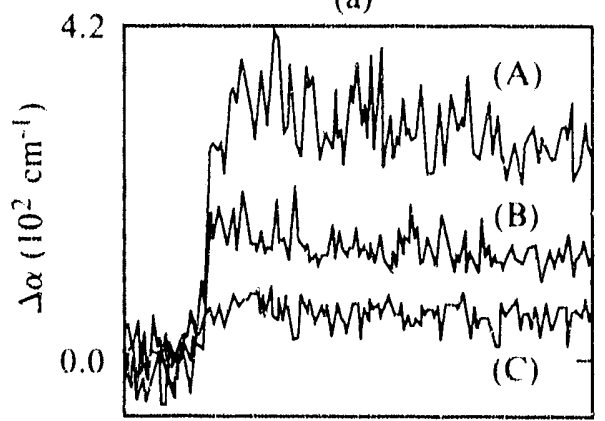

(b)

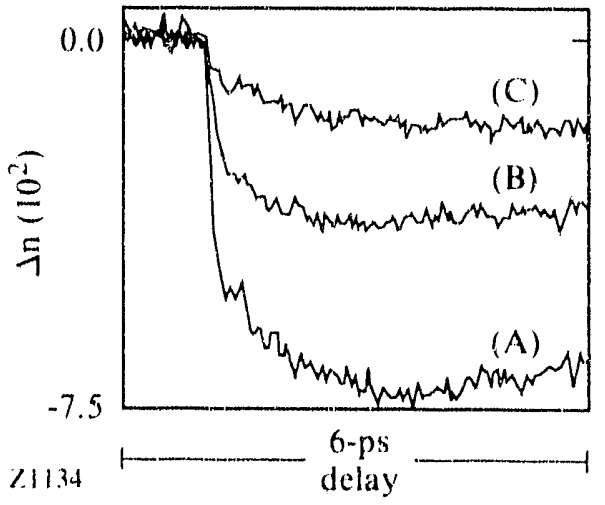

4. Band-Edge Gain Dynamics

For high injected-carrier densities $\left(N>10^{18} \mathrm{~cm}^{-3}\right)$, the combination of nearly instantaneous intra- $\Gamma$-valley redistribution of electrons 12,23 with the rapid scattering of the high-energy $\Gamma$-valley electrons to the $X$ and $L$ valleys ${ }^{10}$ shoulc' make it pessible to observe band-edge gain on subpicosecond time seales even for an excitation at $2 \mathrm{eV}$. Figure 50.30 shows the absorption coefficient measured at $880 \mathrm{~nm}$ for various carrier densities. It is clear that $\alpha$ becomes negative on a subpicosecond time scale for high carrier densities. In fact, gain is observed in a wide spectral region $(850 .-9(0) \mathrm{nm}$ ) on subpicosecond and picosecond time scales for $N>3 \times 10^{16} \mathrm{~cm}^{-314}$ The time delay for gain to occur is $28(0+80)$ fs at $880 \mathrm{~nm}, 450 \pm 1.50 \mathrm{fs}$ at $860 \mathrm{~nm}$, and $65(10 \pm 2(0)$ fs at $850 \mathrm{~nm}$ for the highest carrier density $\left(N \sim 8-10 \times 10^{18} \mathrm{~cm}^{-3}\right)$ used in these measurements. This time increases. $10 \sim 80(4)+30(0)$ fs at 880$) \mathrm{nm}$ and $>30(0) \pm 8(0)$ fs at $860 \mathrm{~nm}$ for lower carrier density $\left.(N-3.3 \times 10)^{1 \mathrm{x}} \mathrm{cm}^{-3}\right)$.

At a probe frequency (1), gain should be observed at any given time when the condition

$$
h(1)<\mu_{\mathrm{eff}}+E_{\mathrm{g}}
$$

with 


$$
\begin{gathered}
\mu_{\mathrm{cfl}}=\frac{m_{e^{\prime}}+m_{h}}{\beta_{\ell^{\prime}} m_{h}+\beta_{h^{\prime} l_{l^{\prime}}}}\left(\beta_{c^{\prime}} \mu_{c^{\prime}}+\beta_{h} \mu_{h}\right) \\
\beta_{e^{\prime}(h)}=\frac{1}{k_{B} T_{e(h)}}
\end{gathered}
$$

is satisfied. Here $\mu_{\ell^{\prime}(h)}$ is the yuasi-chemical potential of the $\Gamma$-valley electrons (holes) with respect to the conduction (valence) band edge, and $E_{g}$ is the normalized band gap. In our model the Coulombenhancement factor is neglected because of the strong plasma screening at these high carrier densities. ${ }^{11,29}$ The calculation of $\mu_{\mathrm{eff}}$ involves solving a set of kinetic equations for the density and kinetic energy of the $\Gamma$-valley electrons and holes. ${ }^{10,14}$ Electrons and holes are assumed to equilibrate instantaneously. The initial temperatures of the electrons and holes are $\sim 3000 \mathrm{~K}$ and $\sim 600 \mathrm{~K}$, respectively, based on the kinetic energies of carriers with $2-\mathrm{eV}$ excitation. Scattering to the $X$ and $L$ valleys is incorporated into the kinetic model as an energy-dependent sink for the high-energy $\Gamma$-valley electrons, again using the effective IDP summarized by Zollner e't al. ${ }^{2.3}$ Zonecenter, LO-phonon emission is also neglected because only a very small amount of energy will be lost by electrons on a subpicosecond time scale when screening is included at high carrier densities. ${ }^{30}$ We have compared the calculated delay times for gain to occur using Eys. (4)-(6) with those deduced from measurements. The hole temperature is treated as a parameter. Good agreement is obtained between model and measurements. 14

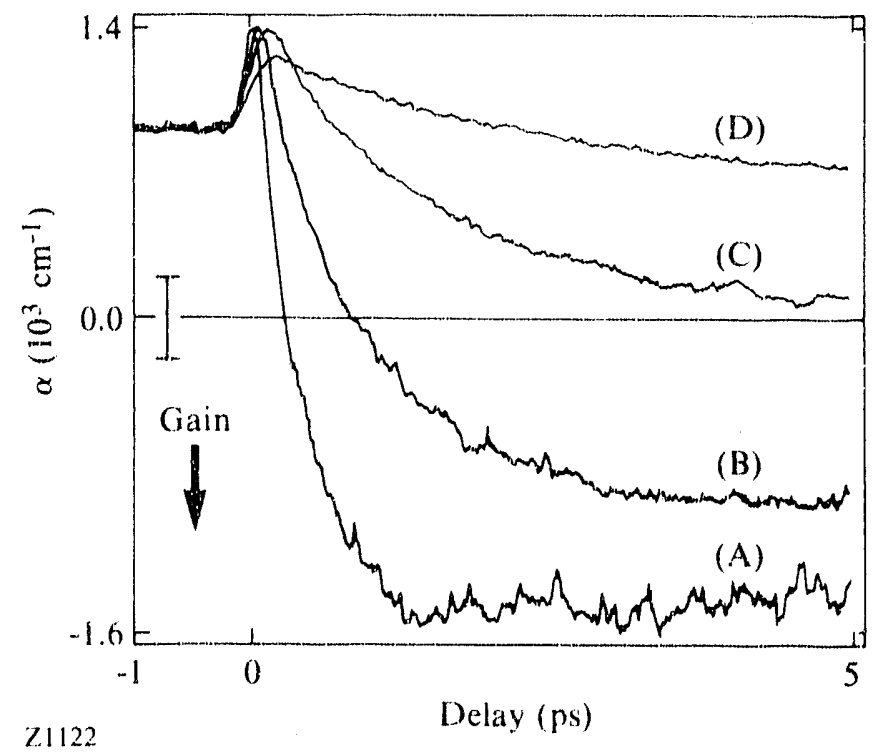

Fig. 50.30)

Measured temporal evolution of the absorption coefficient $\alpha(t)$ at $880 \mathrm{~nm}$ for different nominal $N \sim:$ (A) $9.0 \times 10^{18} \mathrm{~cm}^{-3}$. (B) $3.3 \times 10^{18} \mathrm{~cm}^{-3}$, (C) $1.0 \times 10^{18} \mathrm{~cm}^{-3}$, (D) $3.3 \times$ $1017 \mathrm{~cm}^{-3}$. The error bar associated with the uncertainty on the absolute values of $x$ is indicated. 
Fig. .5().31

The hole temperature $T_{h}$ obtained from the "best fit" between the experiment and the model at different time delays. The dashed line indicates the initial $T_{h}$, based on the kinetic energy of the holes with 2-eV excitation.
In addition to revealing the importance of ultrafast equilibration of carriers near the $\Gamma$ point of the Brillouin zone and the efficient cooling mechanism provided by intervalley scattering, these results also give some insights into hothole dynamics. Figure 50.31 displays the hole temperature that gives a best fit to the data. It appears that the hole distribution is heated to $\sim 800 \mathrm{~K}$ within $300 \mathrm{fs}$, and then cools down to $300 \mathrm{~K}$ within $<1 \mathrm{ps}$. A heated hole distribution indicates that Coulomb-mediated, electron-hole scattering is important on this time scale, in particular when the electron distribution is hotter and for high carricrdensities. This result is supported by theoretical calculations including contributions from intervalence and intravalence band scattering processes. 14,31 The rapid "cooling time" of holes that we obtain also agrees well with those measured in $n$-type GaAs using 100-fs time-resolved luminescence. ${ }^{32}$

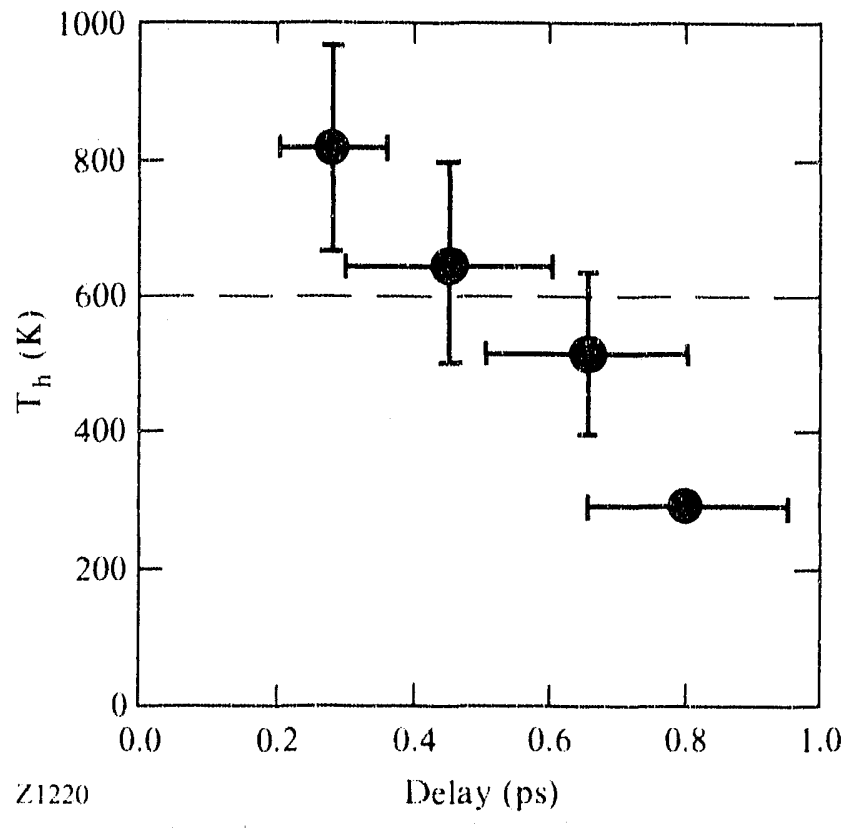

\section{Conclusions}

We have performed a series of measurements on time-resolved absorptive and refractive nonlinearities induced by hot carriers injected at $2 \mathrm{eV}$ on GaAs. Measurements near the initial excited states yield the first observation of refractive-index, spectral-hole burning. Studies of spectral-hole burning and initial scaltering times reveal the importance of intervalley scattering and carriercarrier interactions. Measurements near the band edge show that optical nonlinearities on femtosecond and picosecond time scales are governed by various carrier effects, such as band-gap renormalization, plasma screening, band filling, and free-carrier absorption. Subpicosecond gain near the band edge for high injected-carrier densities demonstrates that the rapid cooling of $\Gamma$-valley electrons is provided by ultrafast intra- $\Gamma$-valley equilibration and intervalley scattering. Evidence of a transient heated-hole distribution provide., insights for the siudy of electron-hole and hole-lattice ineractions. 


\section{ACKNOWLEDGMENT}

We would like to thank J. F. Young and P. J. Kelly at the National Rescarch Council of Canada for performing calculations used in the section on band-edge gain dynamics, and also for many valuable discussions. Contributions from W. L. Nighan, Jr., P. Mert\%, C. Peng, M. Shayegan, and G. W. Wicks are also appreciated. This work was supported by the U.S. Army Reseateh Office under Contact DAAL(13..9) 1-G-(0173, the U.S. Office of Naval Research under Grant N()(0)14-91-J-1488, and the National Science Foundation under Contract ECS-91960())

\section{REFERENCES}

1. C. V. Shank er al., Phys. Rev. Lett. 42, 112 (1979).

2. M. J. Rosker, F. W. Wise, and C. L. Tang, Appl. Phys. Lett. 49, 1726( (986).

3. J. L. Oudar et al., Phys. Rev. Lett. 55, 2074 (1985).

4. J. A. Kash, J. C. Tsang, and J, M. Hvam, Phys. Rev. Lett. 54, 2151 (1985).

5. J. Shath et al., Phys. Rev. Lett. 59, 2222 (1987).

6. W. Z. Lin et al., IEEE J. Quantum Electron. 24, 267 (1988), and references therein.

7. P. C. Becker et al., Phys. Rev. Lett. 61, 1647 (1988).

8. J. A. Kash, Phys. Rev. B 40, 3455 (1989).

9. X. Q. Zhou et al., Solid State Electron. 32, 1591 (1989).

10. D. Kim and P. Y. Yu, Phys. Rev. Lett. 64, 946 (1990).

11. T. Gong, W. L. Nighan, Jr., and P. M. Fauchet, Appl. Phys. Lett. 57, 2713 $(1990)$

12. T. Elsaesser et al., Phys, Rev. Letl. 66, 1757 (1991).

13. T. Gong, P. Mertz. W. L. Nighan, Jr., and P. M. Fauchel, Appl. Phys. Lell. 59. 721 (1991).

14. T. Gong, P. M. Fauchel, J. F. Young, and P. J. Kelly, Phys. Rev. B 44, 6542 (1991).

15. E. Yablonovitch et al., Appl. Phys. Lett. 51, 2222 (1987).

16. C. H. Brito-Cruz, R. L. Fork, and C. V. Shank, in the XV International Comference on Quantum Electronics Technical Digest Series 1987 (Optical Society of America, Washington, DC, 1987), Vol, 21, p. 82.

17. J.-Y. Bigot et al., Phys. Rev. Lett. 65, 3429 (1990).

18. D. Hulin (private communication).

19. T. Gong and P. M. Fauchet (unpublished).

20. G. Bohne et al., in the Technical Digest of the VIIth International Symposium of Ultrafast Processes in Spectroscopy, Bayreuth, Germany, 7-10 October 1991.

21. P. C. Becker et al., Appl. Phys. Lett. 53, 2089 (1988).

22. M. A. Alekseev and D. N. Merlin, Phys. Rev. Lett. 65, 274 (1990); J. A. Kash, J. C. Tsang, and R. G. Ulbrich, Phys. Rev. Lett. 65, 275 (1990). 
23. S. Zollner, S. Gopalan, and M. Cardona, Solid State Commun, 76, 877 $(199())$.

24. J. C. T'sang and J. A. Kash, Phys. Rev. B 34, 600)3 (1986).

25. J. S. Blakemore, J. Appl. Phys, 53, R 223 (1982), and references therein.

26. H. C. Casey, Jr. and M. B. Panish, Heterostructure Lasers, Part A: Findemental Principles (Academic Press, San Diego, 1978), p. 175, and references therein.

27. K. Seeger, Semiconductor Physicis (Springer-Verlag. New York, 1973), p. 379 ,

28. B. R. Bennell, R. A. Soref, and J. A. Det Alamo, IEEE J. Quantum Electron. 26, $113(1990)$.

29. H. Haug and S. W. Koch, Phys. Rev. A 39, 1887 (1989).

30. M. A. Osman and D. K. Ferry, Phys. Rev. B 36, 6018 (1987).

31. J. F. Young, P. Kelly, and N. L. Henry, Phys. Rev. B 36, 4535 (1987).

32. X. Q. Zhou and H. Kurz, in the Terhinical Digest of the VIlth Intermational Symposium of Ultrafast Processes in Spertroscopy, Bayreuth, Germany, 7-10 October 1991; and G. M. Gale 't al., ibid. 


\section{Section 2}

NATIONAL LASER USERS FACILITY

NEWS

NLUF activity during the second quarter of FY92 included experiments on GDL for the University of Illinois and target fabrication for the University of Maryland and the University of Florida. OMEGA shots for both Maryland and Florida have been scheduled.

The University of Illinois, in collaboration with H. Elsayed-Ali at LLE, is conducting an AFOSR-funded experiment to study the effect of laser irradiation of weldments. The GDL laser is used to irradiate welded samples, which are then taken back to the University of Illinois where their physical properties are measured. The energy of the GDL laser allows large-area illumination of the weld. This is a continuing experiment and more GDL shot time is anticipated.

Targets for a series of experiments by $\mathbf{H}$. Griem's group at the University of Maryland were fabricated during this quarter by LLE's Target Fabrication Group. These targets are Ne and Ne-seeded $\mathrm{D}_{2}$ gas-filled plastic shells. The shells are overcoated with an aluminum shinethrough barrier layer that also acts as the gas retention barrier. These targets will measure the line shape of hydrogen-like Ne $x$-ray spectra from dense cores.

C. Hooper from the University of Florida is having a series of Ar and Ar-seeded $D_{2}$ gas-filled plastic shells made for an experiment measuring the effect of density on the shape of the Ar x-ray line spectra from ICF cores. This is to be a time-dependent, line--shape measurement using the SPEAXS instrument and a streak camera connected to a flat crystal spectrograph. 


\section{Section 3 \\ LASER SYSTEM REPORT}

\section{A GDL Facility Report}

There were 208 GDL laser shots during the second quarter of FY92. The 69 target shots were taken by an NLUF user from the University of Illinois. The spatial filter needed by LLE's damage-lesting laboratory was completed, and the 26 system shots were used to complete the filter alignment and start UV damage assessment of large-aperture optics. The 113 laser-system shots were used for system maintenance, alignment, repair, and testing.

J. Kelly is managing the GDL refurbishment project, which is projected to begin in June 1992. First, the laser system is to be completed; then a new target chamber will be moved into the target room. This work will be completed in approximately nine months. The old FORTH-based control system will be replaced with a system similar to what will be used on the upgraded OMEGA laser.

The shot summary for the GDL laser this quarter is as follows:

Laser system

Target

Damage testing

26

TOTAL 208 


\section{B OMEGA Facility Report}

The OMEGA system fired a total of 497 shots during the second quarter of FY92. These shots were divided among the driver line, the laser system, software testing, noise testing, and target irradiation.

Installation of the pulse-shaping apparatus was completed in the driver line. Thirty of the 106 laser-system shots were used to measure how a fast rise-time pulse was affected when propagated through the OMEGA system. The measured pulse shape compared well with the shape predicted with the RAINBOW simulation code. A measured driver-line pulse shape was used as input for the propagation simulation.

During the pulse-shape measurements, it was found that the system radiated a great deal of noisc that caused false triggering of timing electronics in the driver line. The 172 noise test shots were used to check each of the OMEGA pulseforming networks. It was found that there was arcing from the body of the ignitron to ground. In some cases, this arcing was severe enough to cause spallation of material from the ground plane attachment of the ignitron. All of the 56 system ignitrons were remounted to remedy this problem.

The shot summary for the OMEGA laser this quarter is as follows:

Driver line 193

Laser system 106

Software test 21

Noise test 172

Target

\section{ACKNOWLEDGMENT}

This work was supported by the U.S. Department of Energy Office of inertial Confinement Fusion under agreement No. DE-FC03-85DP40200) and by the Laser Fusion Feasibility Project at the Laboratory for Laser Energetics, which is sponsored by the New York State Energy Research and Development Authority and the University of Rochester. 


\section{PUBLICATIONS AND CONFERENCE PRESENTATIONS}

\section{Publications}

S. Alexandrou, R. Sobolewski, and T. Y. Hsiang, "Bend-Induced Even and Odd Modes in Picosecond Electrical Transients Propagated on a Coplanar Waveguide," Appl. Phys. Lell, 60, 1836 (1992).

H. E. Elsayed-Ali, J. W. Herman, and K. K. Lo, "Picosecond Time-Resolved Electron Diffaction Studies of Laser Heated Metals," in the Proceedings of the" Imernational Conferencer on Lasers '90, edited by D. G. Harris and J. Herbelin (STS Press. McLean, VA, 1991), pp. 14.3-1.52.

P. M. Fauchet, "Applied Optical Diagnostics of Semiconductors," Proc. IEEE 80, 420 (1992) (invited paper).

P. M. Fauchel, T. Gong, P. J. Kelly, and J. F. Young. "Femtosecond Gain Dynamics in Thin GaAs Films," Semicond. Sci. Technol. 7, B 164 (1992).

R. H. Hwang-Schweitzer, R. S. Knox, P. B. Gibbs, and J. Biggins, "Fluorescence Studies of Photoregulation in the chrysophyte Ochromonas danica," in J. Lumin. 51, 99 (1992).

G. G. Luther and C. J. McKinstrie, "Cooperative Instabilities of Counterpropagating Light Waves in Homogeneous Plasma," Phys. Rev. Lett. 68, $1710(1992)$.

R. L. McCrory, J. M. Soures, I. Knauer, S. Letzring, F. J. Marshall, S. Skupsky, W. Seka, C. Verdon, D. Bradley, R. S. Craxion, J. Delettrez, R. Epstein, P. Jaanimagi, R. Keck, T. Kessler, H. Kim, R. Kremens, P. W. McKenty, 
R. Short, and B. Yaakobi, "Direct-Drive Implosion Experiments at the Laboratory for Laser Energetics," in Plasma Physics and Controlled Nuclear Fusion Research 1990) (IAEA, Vienna, 1991), Vol 3, pp. 41-52.

D. D. Meyerhofer, S. Augst, C. I. Moore, and J. Peatross, "Aligular Distribution of High-Order Harmonics Generated in the Tunneling Regime," in UltrashortWarelength Lasers (SPIE, Bellingham, WA, 1991), Vol. 1551. pp. 246-251 (invited paper).

W. Seka, R. S. Craxton, R. E. Bahr, D. L. Brown, D. K. Bradley, P. A. Jaanimagi, B. Yalakobi, and R. Epstein, "Production and Characterization of Hot, Long-Scale-Length Laser Plasmas," Phys. Fluids B 4, 432 (1992).

L. J. Shaw-Klein, T. K. Hatwar, S. J. Burns, S. D. Jacobs, and J. C. Lambropoulos, "Anisotropic Thermal Conductivity of Rare Earth-Transition Metal Thin Films," J. Mater. Res. 7, 329 (1992).

\section{Forthcoming Publications}

S. Alexandrou, R. Sobolewski, and T. Y. Hsiang, "Time-Domain Characterization of Bent Coplanar Waveguides," to be published in the IEEE Journal of Quantum Electronicis.

T. R. Boehly, R. S. Craxton, R. J. Hutchison, J. H. Kelly, T. J. Kessler, S. A. Kumpan, S. A. Letzring, R. L. McCrory, S. F. B. Morse, W. Seka, S. Skupsky, J. M. Soures, and C. P. Verdon. "The Upgrade to the OMEGA Laser System," to be published in the Proceedings off SPIE's OE/LASE, Los Angeles, CA, 20-25 January 1992.

D. K. Bradley, J. A. Delettrez, and C. P. Verdon, "Measurements of the Effect of Laser Beam Smoothing on Direct-Drive ICF Capsule Implosions," to be published in Physical Re'view Letters.

D. K. Bradley, P. M. Bell, J. D. Kilkenny, R. Hanks, O. Landen, P. A. Jaanimagi, P. W. McKenty, and C. P. Verdon, "High-Speed Gated X-Ray Imaging for ICF Target Experiments," to be published in the Re'vie' of Scientific Instruments.

P. C. Cheng, H. Kim, and T. H. Lin, "The Study of Silica Deposition in the Leal" Blade of Zea mays $\mathrm{L}$. by X-Ray Contact Microradiography and Confocal Microscopy," to be published in X-Ray Microscropy III.

S. Y. Chou, Y. Liu, W. Khalil, M. I. Nathan, T. Y. Hsiang, and S. Alexandrou, "Ultrafast Nanoscale Metal-Semiconductor-Metal Photodetectors on Bulk and Low-Temperature-Grown GaAs," to be published in Applied Physics Letters.

W. R. Donaldson and L. Mu. "The Effects of Doping on Photoconductive Switches as Determined by Electro-Optic Imaging." to be published in the Proceedings of SPIE's OE/LASE, Los Angeles, CA, 2()-25 January 1992.

E. M. Epperlein, "Laser Filamentation in Plasmas," to be published in the procesedings of the Topical Conference on Research Trends in Inertial Confinement Fusion, La Jolla, CA, 4 6 February 1991.

E. M. Epperlein and R. W. Short, "Nonlocal Heat Transport Effects on the Filamentation of Light in Plasmas," to be published in Physics of Fluids $B$. 
P. M. Fauchet, D. Hulin, A. Mourchid, and R. Vanderhaghen, "Ultrafast Thermal Nonlinearities in Amorphous Silicon," to be published in Ultrafast Laser Probe Phenomena in Semiconductors and Superconductors.

P. M. Fauchet, "Picosecond Spectroscopy in Solids with a Free-Electron Laser," to be published in Spectrosctopic Characterization Tecthiques for Semiconductor Techology $N$ (invited paper).

T. Gong, P. M. Fauchet, J, P. Y'oung, and P. J. Kelly, "Subpicosecond Gain Dynamics in GaAs," to be published in the Preceedings of the 1992 March Mecting of the American Physic al Society, Indianapolis, IN, 16-20 March 1992.

T. Gong, K. B. Ucer. L. X. Zheng, G. W. Wicks, J. F. Young, P. J. Kelly, and P. M. Fauchet, "Femtosecond Carrier-Carrier Interactions in GaAs," to be published in the Procedings of the Eighth International Conference on Ultrafast Phenomena. Antibes-Juan-Les-Pins, France, 8-12 June 1992.

T. Gong and P. M. Fauchet. "Femtosecond Nonlinearities and Hot-Carrier Dynamics in GaAs." to be published in the Proceedings of the VIth International Symposium on Ultafast Processes in Spectroscopy. Bayreuth. Germany. 7-11 October 1991 (invited paper).

M.J. Guardalben and N. George. "Speckle Observation of Pulsed Laser-Induced Dynamics in a Guest-Host Smectic-A Liquiid-Crystal System," to be published in Liquid Crystals.

D. Gupta, W. R. Donaltison, K. Kortkamp, and A. M. Kadin, "Optically Activated Opening Switches." to be published in the Proieedings of SPIE's OE/LASE, Los Angeles. CA, 20-25 January 1992.

J. W. Herman and H. E. Elsayed.Ali, "Time-Resolved Study of Surface Disordering of $\mathrm{Pb}(110)$," to be published in Phisical Reriew Letters.

J. H. Kelly, M. J. Shoup III. M. D. Skeldon, and S. T. Bui, "Design and Energy Characterization of a Multi-Segment Glass Disk Amplifier," to be published in the Proceedings of SPIE's OE/LASE, Los Angeles, CA, 20-25 January 1991.

J. H. Kelly. M. J. Shoup III, M. M. Tedrow, C. D. Kiikka, T. J. Kessler, S. A. Kumpan, A. W. Schmid, M. D. Skeldon, and D. J. Smith, "The $30 \mathrm{~kJ}$ OMEGA Upgrade at the University of Rochester: a Flexible, High-Performance Nd:Glass Driver." to be published in the Procesdings of the IAEA Technical Committe Me'eting on Drivers for Inertial Confinement Fusion, Osaka, Japan. 15-19 April 1991.

J. H. Kelly, M. J. Shoup III, and M. M. Tedrow, "The Effect of lonic and Particulate Platinum on the Performance of Large-Aperture Nd:Phosphate Glass Rod Amplifiers," to be published in the Proceedings of SPIE"SOELASE, LOs Angeles, CA. 20-25 January 1992.

J. H. Kelly, M. J. Shoup III, M. M. Tedrow, and K. Thorp, "Energy Transport in a Modern Disk Amplifier," to be published in the Proceedings of SPIE"s OE/LASE, Los Angeles. CA, 20-25 January 1992.

J. H. Kelly. "Instrumentation Integration in Large Sy'stems: the OMEGA Laser Upgrade at the University of Rochester as an Example," to be published in the 
Procededings of the OSA 1991 Anmul Meetme. San Jese, CA, 3-8 November 1991 (inviled paper).

T. J. Kessler, "Phase Comversion of Brodband Frequency-Tripled Laser Light," to be published in the Procecedings of SPIE"x OE/LASF, Los Angeles. CA, 20).25 January 1992.

H. Kim, B. Yaakobi, J. M. Soures, and P.-C. Cheng. "Latser-Produced Plasma as a Source for X-Ray Microscopy," to be published in X-Ray Microscopy III.

B. S. W. Kuo, J. C. M. Li. and A. W. Schmid, "Thermal Conductivity and Interface Thermal Resistance of Si Film on Si Substrate Determined by Photothermal Displacemem Interferometry," to be published in Applied Physics A.

Y. Lin, W. Seka, J. H. Eberly, H. Huang, and D. L. Brown, "Experimental Investigation of Bessel Beam Characteristics," to be published in Applied Optics (Lasers and Photonics).

Y. Lin and T.J. Kessler, "Raman Scattering in Air: A Four-Dimensional System Analysis." to be published in the Proceed dings of SPIE"s OL:LASE, Los Angeles, CA. 2(1)-25 January 1992.

G. G. Luther and C. J. McKinstrie, "The Transverse Modulational Instability of Counterpropagating Light Waves," to be publi hed in the Irumal of the Optrical Soriety of America $B$.

F. J. Marshall, J. G. Jernigan, T. Collins, J. F. Arens, and G. Pien, "Measuring Laser-Plasma X-Ray Emission Using Pholodiode Arrays," 10 be published in the Rerien of Scientific Instruments.

P. S. Maruthi Sai, M. J. Levene. T. Gillbro. R. S. Knox. R. H. Hwang-Schweityer, and $S$. Mahajan. "Time-Resolved Fluorescence of PhycoerythrocyaninContaining Phycobilisomes from the Cyanobacterium Westicllopsis Prolifica." to be published in the Biophysical Iontrnal

R. L. McCrory, J. M. Soures, J. P. Knauer, S. A. Letering, F. J. Marshall, S. Skupsky, W. Seka, C. P. Verdon, D. K. Bradley, R. S. Craxton, J. A. Deleure", R. Epstein. P. A. Jaanmagi, R. L. Keck, T. J. Kessler, H. Kim. R. I. Kremens, P. W. MoKenty, R. W. Shon, and B. Yaakobi, "Short-Wavelength-Laser Requirements for Direct-Drive Ignition and Gain." 10 be published in the Proceedings of the IAEA Tedmical Committee Mecting on I Drivers for Inertial Confinement Fusion, Osaka. Japan, 15-19 April 1991. and in Lase' and Parricle Berams.

R. L. McCrory, R. E. Bahr, D. K. Bradley, D. L. Brown, R. S. Craxton, J. Delettre\%, R. Epstein, P. A. Sannimagi. T. Kessler. J. P. Knatuer, S. Letzring, F. Marshall. P. W. McKenty. W. Seka. S. Skupsky, J. M. Souves. C. P. Verdon. and B. Yaakobi, "Direct-Drive Implosion Experiments for Laser Fusion on OMEGA and the OMEGA Uperade," to be published in the Prowe cedings of the

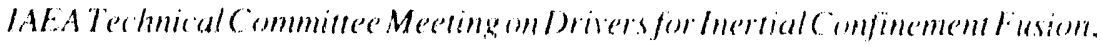
Osaka, Japan, 15-19 April 1991, and in Laser and Parricle Beams.

R. L. McCrory, "Computer Modeling and Simulation in Inertial Confinement Fusion." to be published in /I Nuen'o Cimentu. 
R. L. McCrory, "Laser-Driven ICF Experiments," to be published in Nuclear Fusion by Inertial Confinement.

R. L. McCrory, "Direct-Drive Implosion Experiments for Laser Fusion on OMEGA and the OMEGA Upgrade," to be published in the Procecedings of the 21st ECLIM, Warsaw. Poland, 21-25 October 1991.

R. L.. McCrory, "Irradiation Uniformity Requirements for Direct-Drive Laser Fusion," to be published in the SPIE Proceedings of the XIV International Conference on Coherent and Nonlinear Opsics, St. Petersburg, Russia, 24-27 September 1991.

C. J. McKinstrie and R. Bingham, "Stimulated Raman Forward Scattering and the Relativistic Modulational Instability of Light Waves in Rarefied Plasma," to be published in Physics of Fluids $B$.

C. J. McKinstrie and M. V. Goldman, "Three-Dimensional Instabilities of Counterpropagating Light Waves in Homogeneous Plasmas," to be published in the Journal of the Optical Society of America B.

\section{Conference Presentations}

The following presentations were made at SPIE's OE/LASE, Los Angeles, CA, 20-25 January 1992:

T. R. Boehly, R. S. Craxton, R. J. Hutchison, J. H. Kelly, T. J. Kesster, S. A. Kumpan, S. A. Letzring, R. L. McCrory, S. F. B. Morse, W. Seka, S. Skupsky, J. M. Soures, and C. P. Verdon. "The Upgrade to the OMEGA Laser System."

W. R. Donaldson and L. Mu, "The Effects of Doping on Photoconductive Switches as Determined by Electro-Optic Imaging."

D. Gupta. W. R. Donaldson. K. Kortkamp, and A. M. Kadin, "Optically" Activated Opening Switches."

J. H. Kelly, M. J. Shoup III. and M. M. Tedrow, "The Effect of lonic and Particulate Platinum on the Performance of Large-Aperture Nd:Phosphate Glass Rod Amplifiers."

J. H. Kelly, M. J. Shoup III, M. M. Tedrow, and K. Thorp, "Energy Transport in a Modern Disk Amplifier."

T. J. Kessler, "Phase Conversion of Broadband Frequency-Tripled Laser Light."

Y. Lin and T. J. Kessler, "Raman Scattering in Air: A Four-Dimensional System Analysis."

M. J. Shoup III. J. H. Kelly, M. M. Tedrow, F. A. Rister, and K. Thorp. "Mechanical Design of 15- and 20-cm Clear Aperture Disk Amplifiers for the OMEGA Upgrade."

M. J. Shoup III, S. D. Jacobs, A. H. Kelly, C. T. Cotton, S. F. B. Morse, and S. A. Kumpan, "Specification of Large-Aperture Nd:Phosphate Glass Laser Disks."

M. D. Skeldon. S. Bui, S. A. Letzring, and W. Siryk, "Implementation of Pulse Shaping on the OMEEGA Laser System." 
M. M. Tedrow, J. H. Kelly, M. J. Shoup III, R. Juhala, A. Reynolds, L. Allen, and G. Dubé, "Characterization of a Diode-Pumped, 3.8-cm Clear-Aperture. HighGain, Active-Mirror Laser Amplifier Using Cr:Nd:GSGG and Nd:GGG," presented at the Seventh Topical Meeting on Advanced Solid-State Lasers, Santat Fe, NM, 17-19 February 1992.

The following presentations were made at the Ninth Topical Conference on High Temperature Plasma Diagnostics, Santa Fe, NM, 15-19 March 1992:

D. K. Bradley, "High-Speed Gated X-Ray Imaging for ICF Targa Experiments" (invited paper).

F. J. Marshall, J. G. Jernigan, T. Collins, J. F. Arens, and G. Pien, "Measuring Laser-Plasma X-Ray Emission Using Photodiode Arrays."

The following presentations were made at the American Physical Society, Indianapolis, IN. 16-20 March 1992:

H. E. Elsayed-Ali, "Time-Resolved Reflection High-Energy Electron Diffraction Studies of Laser-Heated Metal Surfaces" (invited talk).

P. M. Fauchet, T. Gong, Y. Kostoulas, K. B. Ucer, and G. W. Weeks, "Femtosecond Spectral Hole Burning in III-V Semiconductors."

T. Gong, P. M. Faucher, J. P. Young, and P. J. Kelly, "Subpicosecond Gain Dynamics in GaAs."

J. W. Herman and H. E. Elsayed-Ali, "Time-Resolved Study of the Surface Structure of $\mathrm{Pb}(110)$ and $\mathrm{Pb}(111), "$

The following presentations were made at SPIE's 1992 Symposium on Compound Semiconductor Physicsi and Devices, Somerset, NJ. 22-26 March 1992:

P. M. Fauchet and T. Gong, "Femtosecond Dynamics of Hot Carriers in GaAs."

P. M. Fauchet, D. Hulin. A. Mourchid, and R. Vanderhaghen, "Ultrafast Thermal Nonlinearities in Amorphous Silicon."

P. M. Fauchet, "Picosecond Spectroscopy in Solids with a Free-Electron Laser" (invited paper).

\section{ACKNOWLEDGMENT}

The work described in this volume includes current research at the Laboratory for Lawer Energetics, which is supported by New York State Energy Research and Development Authority, the University of Rochester, the U.S. Department of Energy Office of Inertial Confinement Fusion under agreement No. DE-FCO3-85DP40200, and other agencies. 

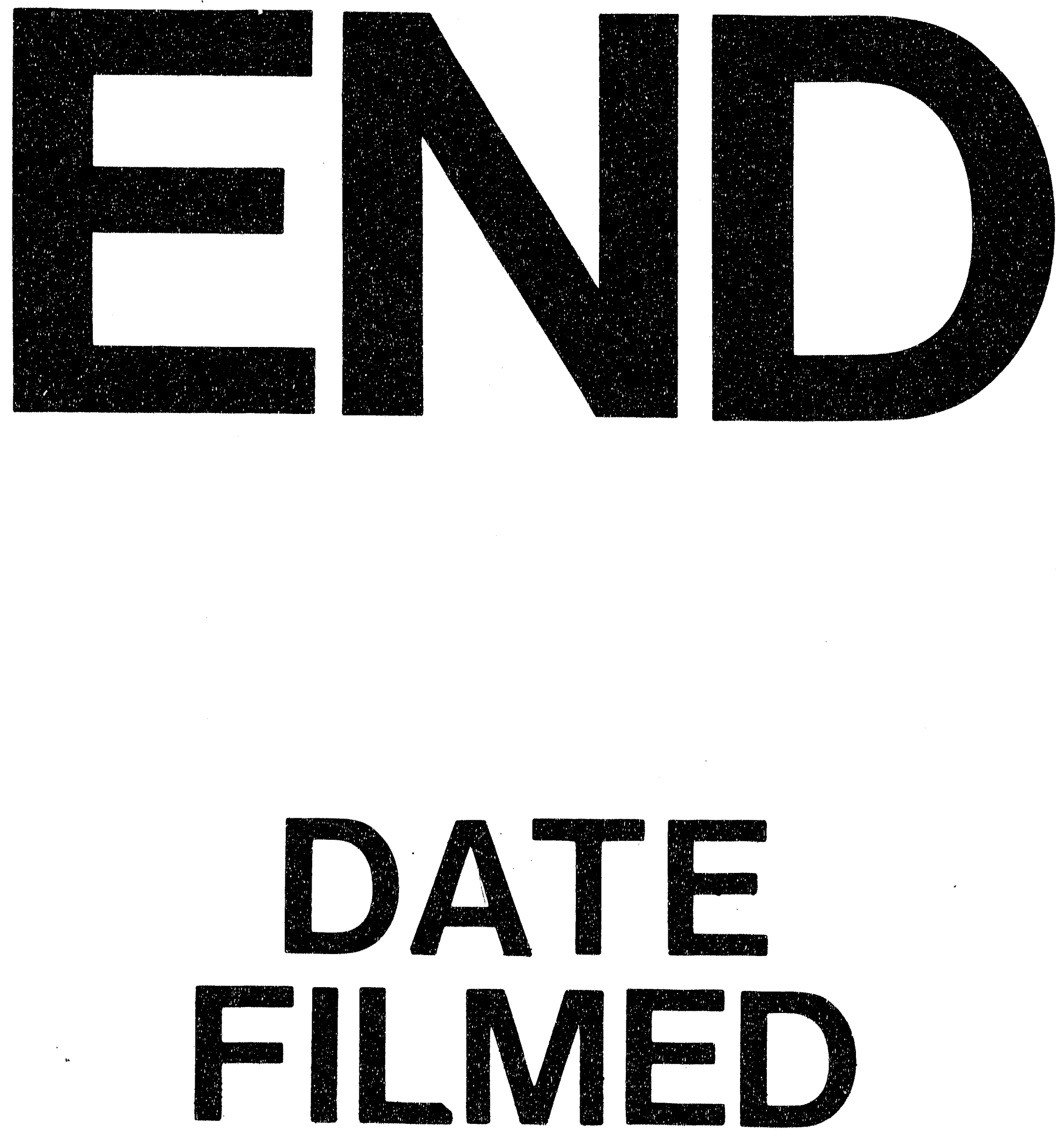

桪

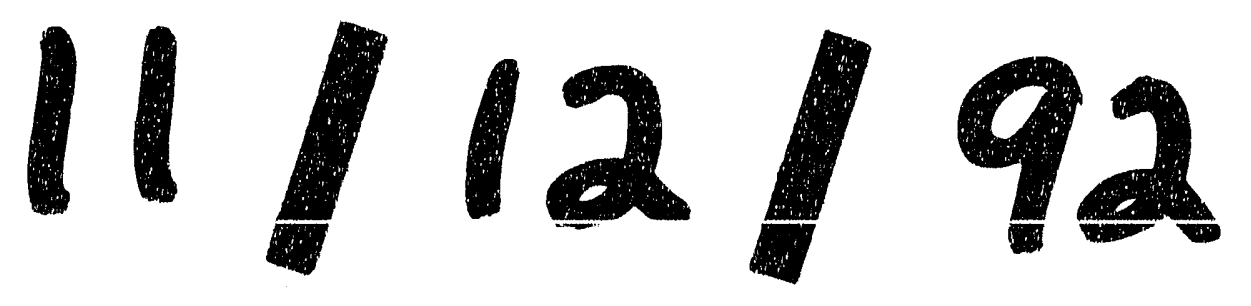


